\title{
Fractional Quantum Hall States of Clustered Composite Fermions
}

\author{
Arkadiusz Wójs ${ }^{1,2}$, Kyung-Soo Yi ${ }^{1,3}$, and John J. Quinn ${ }^{1}$ \\ ${ }^{1}$ University of Tennessee, Knoxville, Tennessee 37996, USA \\ ${ }^{2}$ Wroclaw University of Technology, 50-370 Wroclaw, Poland \\ ${ }^{3}$ Pusan National University, Pusan 609-735, Korea
}

\begin{abstract}
The energy spectra and wavefunctions of up to 14 interacting quasielectrons (QE's) in the Laughlin $\nu=\frac{1}{3}$ fractional quantum Hall (FQH) state are investigated using exact numerical diagonalization. It is shown that at sufficiently high density the QE's form pairs or larger clusters. This behavior, opposite to Laughlin correlations, invalidates the (sometimes invoked) reapplication of the composite fermion picture to the individual QE's. The series of finite-size incompressible ground states are identified at the QE filling factors $\nu_{\mathrm{QE}}=\frac{1}{2}, \frac{1}{3}, \frac{2}{3}$, corresponding to the electron fillings $\nu=\frac{3}{8}, \frac{4}{11}$, $\frac{5}{13}$. The equivalent quasihole $(\mathrm{QH})$ states occur at $\nu_{\mathrm{QH}}=\frac{1}{4}, \frac{1}{5}, \frac{2}{7}$, corresponding to $\nu=\frac{3}{10}, \frac{4}{13}, \frac{5}{17}$. All these six novel FQH states were recently discovered experimentally. Detailed analysis indicates that $\mathrm{QE}$ or $\mathrm{QH}$ correlations in these states are different from those of well-known FQH electron states (e.g., Laughlin or Moore-Read states), leaving the origin of their incompressibility uncertain. Halperin's idea of Laughlin states of QP pairs is also explored, but is does not seem adequate.
\end{abstract}

PACS numbers: 71.10.Pm, 73.43.-f

\section{INTRODUCTION}

Pan et al $\stackrel{1}{1}$ recently (and Goldman and Shayegan ${ }^{2}$ a little earlier) observed fractional quantum Hall (FQH) effect ${ }^{3.4}$ in a two-dimensional electron gas at novel filling fractions $\nu$ of the lowest Landau level $\left(\mathrm{LL}_{0}\right)$. The new FQH states are found to be spin-polarized and occur between the neighboring $\nu=\frac{1}{3}$ and $\frac{2}{5}$ states of the Jain sequence,,$\frac{5}{,}$ corresponding to one and two completely filled composite fermion (CF) LL's, respectively. Some of them, such as $\nu=\frac{4}{11}$ or $\frac{4}{13}, 2$ appear in the standard hierarchy of quasiparticle (QP) condensates formulated by Haldane ${ }^{6}$ and Halperin ${ }^{7}$ but their "hierarchical" interpretation was earlier ruled out $\underline{\underline{8}}$ because of the specific (subharmonic) form of the QP-QP interaction. Others, such as the $\nu=\frac{3}{8}$ or $\frac{3}{10}$ states, do not belong to the Haldane hierarchy, and the origin of their incompressibility is puzzling in an even more obvious way.

Recently there have been attempts $\frac{9}{}$ to explain these states in terms of "multi-flavor" CF pictures, with coexisting CF's carrying different numbers of fluxes (vortices). Being equivalent to the $\mathrm{CF}$ hierarchy 10 of Laughlincorrelated spin-polarized QP states, not only are these models not conceptually new, but they are also unjustified at the QP fillings in question $\stackrel{8}{-}$ In fact, some of the numerical results obtained earlier in a slightly different context ${ }^{11.12}$ and more detailed results presented here show that they do not describe the new FQH states.

The appeal of the CF models lies in the fact that they allow one to think of a more complicated FQH state at filling factor $\nu$ as a $\mathrm{CF}$-analogue of a simpler and better understood state at an effective CF filling factor $\nu^{*}$. The best known examples are $\nu=\frac{1}{3}$ and $\frac{2}{5}$ states corresponding to $\nu^{*}=1$ and 2 , respectively. In the present case $\nu=\frac{4}{11}$ and $\frac{3}{8}$ correspond to $\nu^{*}=\frac{4}{3}$ and $\frac{3}{2}$, that is to onethird and one-half filled first excited CF LL (CF-LL 1$)$, respectively. However, it cannot come as a surprise that the $\mathrm{CF}$ model does not always work. It is valid only for systems which support Laughlin correlations, and those only occur if the interactions are sufficiently strong at short range 13 Moreover, it is known precisely that "sufficiently strong" means that the interaction pseudopotential is superharmonic ${ }^{11,12}$ It should also be noted that the $\mathrm{CF}$ analogy is not sufficient to explain all new observed states regardless of the fact that the (reapplied) CF model does not $t^{\frac{8}{2}}$ generally describe correlations between the CF's themselves. Specifically, the $\nu=\frac{3}{10}$ state corresponds to $\nu^{*}=\frac{3}{4}$, while the electronic state at this filling is not incompressible.

The aim of this paper is to present the results of our "numerical experiments" for the new FQH states and show that they are described by grouping of QP's into pairs 14? (although probably without pair-pair Laughlin correlations 16 ) or into larger clusters 17 rather than by a multi-flavor $\mathrm{CF}$ picture. To do so, we: (i) present results of extensive numerical diagonalization studies of up to 14 interacting Laughlin quasielectrons (QE's); (ii) demonstrate directly the QE clustering by the calculation of pair amplitudes; (iii) identify the series of finitesize nondegenerate ground states at $\nu_{\mathrm{QE}}=\frac{1}{2}, \frac{1}{3}, \frac{2}{3}$, corresponding to $\nu=\frac{3}{8}, \frac{4}{11}, \frac{5}{13}$; (iv) investigate the dependence of the stability of these states on the details of the QE-QE interaction pseudopotential; (v) calculate their pair-correlation functions; (vi) show that they have different QE-QE correlations than those of electrons in the Laughlin ${ }^{4}$ or Moore-Read 18.19 .20 states; (vii) construct the equivalent quasihole $(\mathrm{QH})$ states at $\nu_{\mathrm{QH}}=\frac{1}{4}$, $\frac{1}{5}, \frac{2}{7}$, corresponding to $\nu=\frac{3}{10}, \frac{4}{13}, \frac{5}{17}$; (viii) discuss a recent mode 14 assuming pairing of QP's and Laughlin correlations between the pairs (originally proposed by Halperin ${ }^{16}$ for electrons in $\mathrm{LL}_{0}$ ); and (ix) propose an explanation for the FQH state observed at $\nu=\frac{6}{17}$.

Standard numerical calculations for $N_{e}$ electrons are not useful for studying the new states, because convincing results require too large values of $N_{e}$. Therefore, we 
take advantage of the knowledge $e^{8,21,22,23}$ of the dominant features of the pseudopotential $V_{\mathrm{QE}}(\mathcal{R})$ of the $\mathrm{QE}-\mathrm{QE}$ interaction (i.e., the $\mathrm{QE}-\mathrm{QE}$ interaction energy $V_{\mathrm{QE}}$ as a function of relative pair angular momentum $\mathcal{R}$ ), and diagonalize the (much smaller) interaction Hamiltonian of the $N$-QE systems. This procedure was earlier shown ${ }^{21}$ to accurately reproduce the low-energy $N_{e}$-electron spectra at filling factors $\nu$ between $\frac{1}{3}$ and $\frac{2}{5}$. It was also used in a similar, many-QE calculation by Lee et al ${ }^{23}$ (who, however, have not found support for QE clustering).

Our results confirm series of nondegenerate ground states with finite excitation gaps at $2 l$, twice the QE angular momentum, equal to $2 N-3,3 N-7$, and $\frac{3}{2} N+2$ (the last two states are particle-hole symmetric). These series extrapolate to $\nu_{\mathrm{QE}} \equiv N /(2 l+1)=\frac{1}{2}, \frac{1}{3}$, and $\frac{2}{3}$, and to the electron filling factors $\nu=\frac{3}{8}, \frac{4}{11}$, and $\frac{5}{13}$, respectively. The fact that the $\nu_{\mathrm{QE}}=\frac{1}{3}$ sequence occurs at $2 l=$ $3 N-7$ rather than $3 N-3$ implies that this state is not a Laughlin state of QE's (or CF's). Indeed, the assumption that the $\nu_{\mathrm{QE}}=\frac{1}{3}$ sequence must be described by the relation $2 l=3 N-3$ led to its being overlooked in earlier finite-size calculations ${ }^{24}$ The identified sequence is also different from $2 l=3 N-5$ characteristic of Halperin's paired state $\frac{16}{\underline{n}}$ corresponding to $\nu_{\mathrm{QE}}=\frac{1}{3}$. On the other hand, the value of $2 l=2 N-3$ for the $\nu_{\mathrm{QE}}=\frac{1}{2}$ sequence suggests that this state could be a Halperin paired QE state (Laughlin state of QE pairs) $)^{12,14.16}$ similar to the Moore-Read ${ }^{18,19,20}$ state of electrons at the half-filling of $\mathrm{LL}_{1}$. However, the squared overlaps with the MooreRead state are very small $(\sim 0.03$ for $N \leq 14)$, and the nondegenerate ground states occur in this series only for odd numbers of $\mathrm{QE}$ pairs $\left(\frac{1}{2} N=3,5\right.$, and 7$)$, which implies that the nature of this state is different.

The comparison of the $\mathrm{QH}-\mathrm{QH}$ and $\mathrm{QE}-\mathrm{QE}$ pseudopotentials (which differ mainly by a hard-core at $\mathcal{R}=1$ for the QH's) result in the following correspondence relation for the incompressible QH and QE states

$$
\nu_{\mathrm{QH}}^{-1}=2+\nu_{\mathrm{QE}}^{-1} .
$$

For $\nu_{\mathrm{QE}}=\frac{1}{2}, \frac{1}{3}$, and $\frac{2}{3}$, this relation gives $\nu_{\mathrm{QH}}=\frac{1}{4}, \frac{1}{5}$, and $\frac{2}{7}$, corresponding to $\nu=\frac{3}{10}, \frac{4}{13}$, and $\frac{5}{17}$, respectively, all of which have also been observed experimentally.

To understand the origin of incompressibility in the new states we explore an idea ${ }^{12.14}$ of Laughlin states of QP pairs (originally proposed by Halperin ${ }^{16}$ to describe electron pairing in $\mathrm{LL}_{0}$ ). Grouping of QE's or QH's into pairs or even larger clusters at sufficiently large filling factors can be predicted from the form of $\mathrm{QE}-\mathrm{QE}$ and $\mathrm{QH}-$ $\mathrm{QH}$ pseudopotentials, characterized by strong minima at $\mathcal{R}_{\mathrm{QE}}=1$ state and $\mathcal{R}_{\mathrm{QH}}=3$. It is clearly demonstrated by the calculation of the appropriate pair amplitude coefficients ${ }^{25}$ (related to the fractional grandparentage ${ }^{26}$ ) in the many-QE ground states. In Halperin's paired state, Laughlin correlations between the QP pairs would depend on the superharmonic behavior of the pair-pair interaction pseudopotential, $V_{\mathrm{QP}_{2}}\left(\mathcal{R}_{2}\right)$, at the relevant values of $\mathcal{R}_{2}$, the relative angular momentum of two pairs. The analysis of the calculated $V_{\mathrm{QE}_{2}}\left(\mathcal{R}_{2}\right)$ suggests that of the whole sequence of incompressible Laughlin states of QE pairs, only $\nu_{\mathrm{QE}}=\frac{1}{2}$ might satisfy the condition for Laughlin correlations. This appears to be in agreement with our "numerical experiments," which reveal the $\nu_{\mathrm{QE}}=\frac{1}{2}$ series at $2 l=2 N-3$ (as predicted for Halperin's paired state) and the $\nu_{\mathrm{QE}}=\frac{1}{3}$ series at $2 l=3 N-7$ (different from $3 N-5$ of a Halperin's paired state). However, as mentioned above, we find several strong indications that Halperin's paired state does not occur for QE's at neither $\nu_{\mathrm{QE}}=\frac{1}{2}$ nor $\frac{1}{3}$.

\section{PSEUDOPOTENTIALS, LAUGHLIN CORRELATIONS, AND THE COMPOSITE FERMION PICTURE}

The essential information about the interaction of particles confined to some Hilbert space can be obtained by defining the value of interaction energy for all allowed pair states. For charged particles confined to a LL in the presence of a magnetic field, the relative motion is strongly quantized. The orbital pair eigenstates can be labeled with a single discrete quantum number, relative angular momentum $\mathcal{R}$. This number is a non-negative integer; it must be odd (even) for a pair of identical fermions (bosons), and it increases with increasing average distance $\sqrt{\left\langle r^{2}\right\rangle}$ between the two particles.

In Haldane's spherical geometry,$\underline{6}$ most convenient for finite-size calculations, the $\mathrm{LL}_{0}$ is represented by a degenerate shell of single-particle angular momentum $l=Q$. Here $2 Q \phi_{0}$ is the strength of Dirac monopole in the center, defined as $4 \pi R^{2} B$, the total flux of the magnetic field $B$ through the surface of radius $R$ (using the definition of the magnetic length $\lambda=\sqrt{\hbar c / e B}$, this can be written as $Q \lambda^{2}=R^{2}$ ). The total pair angular momentum $L^{\prime}$ (here, $L$ means total angular momentum of $N$ particles, and $L^{\prime}$ is reserved for $N=2$ ) results from an addition of two angular momenta $l$ of individual particles, and it is connected to the relative pair angular momentum via relation $L^{\prime}=2 l-\mathcal{R}$. Thus, the maximum value of $L^{\prime}=2 l$ (for bosons) or $2 l-1$ (for fermions) corresponds to the smallest pair state with $\mathcal{R}=0$ or 1 .

The pair interaction energy $V$ expressed as a function of $\mathcal{R}$ is called the pseudopotential, and the series of its parameters $V(\mathcal{R})$ entirely determines many-body correlations. On a sphere, $\mathcal{R} \leq 2 l$ and thus the number of pseudopotential parameters is finite. However, even in an infinite (planar) system, only those few leading parameters at the values of $\mathcal{R}$ corresponding to the average distance $\sqrt{\left\langle r^{2}\right\rangle}$ not exceeding the correlation length $\xi$ are of significance (provided that the correlations are indeed characterized by finite $\xi \sim \lambda$ ).

Remarkably, even for the completely repulsive interactions, different correlations can result in a partially filled shell depending on the form of $V(\mathcal{R})$. For example, if $V$ increases as a function of $\mathcal{R}$ (as in atomic shells in the absence of magnetic field), the low-energy many-body 
states obey Hund's rule and tend to have the maximum possible degeneracy (i.e., the maximum $2 L+1$ ). In the opposite extreme situation, when $V$ decreases sufficiently quickly ${ }^{13}$ as a function of $\mathcal{R}$, Laughlin correlations occur. These correlations are defined as the tendency to avoid pair states with one or more smallest values of $\mathcal{R}$, i.e., with the largest repulsion (the relative occupation of different pair states in a many-body state is a well-defined quantity, given by the pair amplitude coefficient ${ }^{25}$ ).

As a result of Laughlin correlations, the low-energy many-body states usually have small degeneracy and effects commonly associated with the FQH physics occur, including the formation of incompressible ground states at certain values of $\nu$. What is often not realized or overlooked is that it is precisely the Laughlin correlations that justify the CF picture. In other words, the mean field $\mathrm{CF}$ picture that attaches $2 p$ magnetic flux quanta (or vortices) to each fermion and predicts the family of Jain wavefunctions for the lowest energy states is correct if and only if those fermions have Laughlin correlations, i.e., the lowest energy states indeed maximally avoid having pair states with $\mathcal{R} \leq(2 p+1)$. For example, in order to bind $2 p$ vortices and transform into CF's, electrons must have Laughlin correlations (and indeed they do in $\mathrm{LL}_{0}$ ). These CF's (or, more precisely, the QP's in partially filled CF-LL's) would bind additional vortices and turn into "higher-order" CF's if they themselves had Laughlin correlations (and in this paper we show that, at the relevant filling factors, they do not).

Another important class of pseudopotentials are the "harmonic" ones, i.e., those for which parameters $V_{\mathrm{H}}(\mathcal{R})$ fall on a straight line when plotted as a function of the average squared distance $\left\langle r^{2}\right\rangle$. Clearly, all harmonic potentials $V_{\mathrm{H}}(r)=a_{0}+a_{2} r^{2}$ have this property regardless of the LL confinement. It has been shown 11 that for particles confined in an angular momentum shell on a sphere, $V_{\mathrm{H}}$ is a linear (increasing in case of repulsion) function of squared pair angular momentum, $L^{\prime}\left(L^{\prime}+1\right)$. It follows from considering the large-radius limit $(R \rightarrow \infty$ and $\lambda=$ const) that on a plane (or on a "large" sphere, i.e., for $\mathcal{R} \ll 2 l$ ), $V_{\mathrm{H}}$ is a linear function of $\mathcal{R}$. The importance of the harmonic pseudopotential lies in the fact that it causes no correlations, i.e., all many-body states with the same total angular momentum $L$ are degenerate (and their energy is just a linear function of $L(L+1)$ or $\mathcal{R}$, depending on geometry) $\stackrel{11}{=}$ It is thus only the anharmonic part of $V(\mathcal{R})$ that causes correlations, while the harmonic part only shifts the entire energy spectrum by a constant times $L(L+1)$ or $\mathcal{R}$.

From the analysis of the sum rules 27 obeyed by the pair amplitudes $\mathcal{G}_{\Psi}(\mathcal{R})$ measuring the fraction of pairs with relative pair angular momentum $\mathcal{R}$ out of the total number of $\frac{1}{2} N(N-1)$ pairs in an $N$-particle state $\Psi$, it has been shown $\frac{11.12}{}$ that Laughlin correlations occur near filling factor $\nu$ if the dominant anharmonic contribution to $V$ is positive at the avoided values of $\mathcal{R}$. For example, for fermions at $\nu \approx(2 p+1)^{-1}=\frac{1}{3}$, the pseudopotential $V(\mathcal{R})$ must decrease "superlinearly" through any three values $a<b<c$ beginning with $a=1$. By the superlinear (i.e., superharmonic) behavior we mean that

$$
\frac{V(a)-V(b)}{b-a}>\frac{V(b)-V(c)}{c-b} .
$$

Only then do Laughlin correlations occur and justify the use of the mean field CF transformation that attaches $2 p=2$ fluxes (vortices) to each electron. Moreover, any pseudopotential that is strongly superharmonic at short range causes the same (Laughlin) correlations which explains the robust character of the FQH states in realistic systems or in model calculations.

It has been shown 11 that it is the superharmonic behavior of the Coulomb repulsion $V(r) \sim r^{-1}$ in $\mathrm{LL}_{0}$ in the entire range of $\mathcal{R}$ that explains the success of the CF picture through the entire Jain sequence of fractions. ${ }^{5}$ It was also shown ${ }^{12}$ (by direct calculation of pair amplitudes) that because the Coulomb pseudopotential in $\mathrm{LL}_{1}$ is roughly linear between $\mathcal{R}=1$ and 5 , the electrons tend to form pairs with $\mathcal{R}=1$ when filling a fraction $\frac{1}{4} \leq \nu_{1} \leq \frac{1}{2}$ of $\mathrm{LL}_{1}$. This is exactly the opposite behavior to the avoidance of this pair state that would characterize a state with Laughlin correlations and that could justify the CF picture (sometimes erroneously used in literature to describe the FQH states at $\nu \equiv 2+\nu_{1}=\frac{5}{2}, \frac{7}{3}$, or $\frac{8}{3}$ ).

Let us stress here that the mean field CF picture simply mimics the fact that (in a Laughlin-correlated system) each electron drags a $\mathcal{R}=1$ correlation hole with it - by replacing the "bare" electron LL degeneracy with an appropriately smaller, "effective" one (and an effective $\mathrm{CF}$ magnetic field $B^{*}$ is just an intuitive physical picture that cannot be treated literally). In fact, it has recently been demonstrated 28 that the adiabatic addition of flux (instead of addition via gauge transformation) automatically gives rise to Laughlin correlations without the need of any mean field approximation. Having said this, there simply are no CF's in the $\nu=\frac{5}{2}, \frac{7}{3}$, or $\frac{8}{3}$ states, let alone the CF pairs. Instead, at least the Moore-Read state at $\nu=\frac{5}{2}$ is clearly a paired state of electrons $s^{12.20}$ (although models involving pairing of CF's in this state can also be found in literature ${ }^{29}$ ). It is surprisingly often overlooked that the FQH effect does not prove the existence of CF's or Laughlin correlations, but only the existence of a nondegenerate ground state separated from the continuum of QP excitations by a finite gap - the property which can also result from correlations of a different nature.

It is indeed quite remarkable that the knowledge of the interaction pseudopotential $V(\mathcal{R})$ at short range is sufficient to predict or rule out Laughlin correlations in different FQH systems ${ }^{8.11 .30 .31}$ However, it must be carefully noticed that the predicted absence of Laughlin correlations does not preclude the FQH effect itself, only a microscopic origin of the effect attributable to Laughlin correlations. It should also be realized that immediate application of the CF model without studying the interactions between the relevant particles (electrons, QP's, etc.) whenever real or numerical experiments reveal incompressibility is not justified. Precisely such a situation 
was recently encountered with the discovery of new FQH states at $\nu=\frac{3}{8}, \frac{4}{11}$, etc., which turn out not to be Laughlin or Jain states (of QP's) despite being incompressible.

\section{QP INTERACTIONS}

It follows from the preceding discussion that in order to explain the origin of incompressibility in the new FQH states, one has to begin with the identification of the relevant (quasi) particles (electrons, holes, Laughlin QP's, CF's, excitons, skyrmions, ...), analyze their interaction pseudopotentials, understand their correlations, and finally derive the filling factors $\nu$ at which those correlations cause incompressibility. In contrast to the $\mathrm{CF}$ model (which, nevertheless, is still very elegant and useful after it is proven valid for a particular system), this line of thought is free of unproven assumptions, such as that of a cancellation between the Coulomb and gauge interactions beyond the mean field.

It is well-established that a (Laughlin-correlated) system of electrons at $\frac{1}{3} \leq \nu \leq \frac{2}{5}$ can be viewed as one of (fractionally charged and thus less strongly interacting) QE's moving in the underlying Laughlin $\nu=\frac{1}{3}$ ground state. This is elegantly pictured in the $\mathrm{CF}$ model, in which the Laughlin state corresponds to the completely filled CF-LL $L_{0}$, and the QE's correspond to the (weakly interacting) particles moving in the (partially filled) CF$\mathrm{LL}_{1}$. Similarly, the electron system at $\frac{1}{4} \leq \nu \leq \frac{1}{3}$ can be viewed as the QH's moving over the $\nu=\frac{1}{3}$ background (with the QH's pictured as vacancies in $\mathrm{CF}-\mathrm{LL}_{0}$ ).

Therefore, we begin the study of the new FQH states in the $\frac{1}{4} \leq \nu \leq \frac{2}{5}$ range with the analysis of the $\mathrm{QE}-\mathrm{QE}$ and $\mathrm{QH}-\mathrm{QH}$ pseudopotentials. In the following we will use the fermionic statistics to describe QP's which is consistent with the CF picture (and conversion to bosons or anyons can be done in a standard way ${ }^{32}$ ). The qualitative behavior of $V_{\mathrm{QP}}(\mathcal{R})$ at short range is well-known from the numerical studies of small systems ${ }^{8.21 .22 .23}$ In Fig. 1(b) we compare $V_{\mathrm{QE}}(\mathcal{R})$ calculated for the systems of $N=8$ to 12 electrons. As the calculation involves subtraction of the $N$-electron energies corresponding to zero, one, and two QE's that (in finite systems) occur at different values of $Q=\sqrt{R / \lambda}$ (i.e., different surface curvatures), the zero of energy is determined much less accurately than the relative values of different pseudopotential parameters. However, when the data for each $\mathcal{R}$ are extrapolated to large $N$, the positive sign of $V_{\mathrm{QE}}(\mathcal{R})$ is restored, as shown in Fig. 1(c). Still, only the relative values are of importance, since adding a constant to $V(\mathcal{R})$ does not affect correlations and only shifts the whole many-body spectrum by a (different) constant. On the other hand, the repulsive character of the $\mathrm{QP}-\mathrm{QP}$ interaction and the long-range behavior of $V_{\mathrm{QP}}(\mathcal{R}) \sim \mathcal{R}^{-1 / 2}$ follow from the fact that QP's are charged particles (the form of QP charge density affects $V_{\mathrm{QP}}$ only at short range, comparable to the QP size). In Fig. [1(d) we plot $V_{\mathrm{QE}}(\mathcal{R})$ obtained more recently by Lee et al $I^{22}$ using a somewhat different

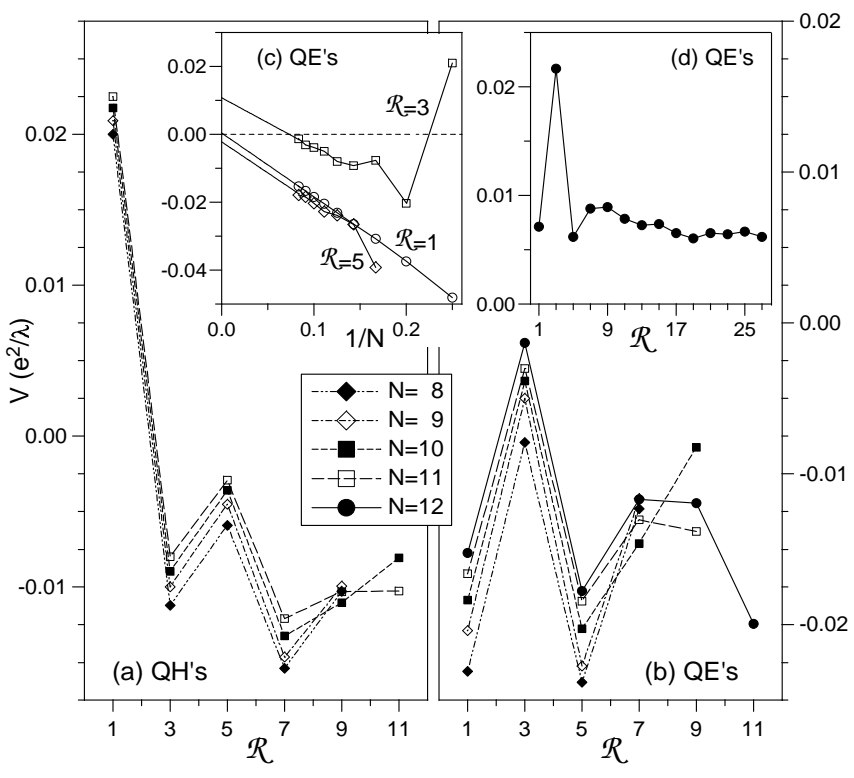

FIG. 1: Interaction pseudopotentials $V(\mathcal{R})$ for the QH's (a) and QE's (b) of the Laughlin $\nu=\frac{1}{3}$ state calculated in the systems of up to $N=12$ electrons on a sphere. Insets: (c) Dependence of the leading QE-QE pseudopotential coefficients corresponding to the smallest values of $\mathcal{R}$ on $N^{-1}$. Extrapolation to $N^{-1} \rightarrow 0$ corresponds to an infinite planar system. (d) QE-QE pseudopotential calculated by Lee et $a l^{22}$

approach. Since it confirms the oscillatory behavior at short range in Fig. 1(b) and behaves as expected at long range, we will use it later to diagonalize interaction in the systems of more than two QE's.

Clearly, the dominant features of $V_{\mathrm{QE}}$ are the small value at $\mathcal{R}=1$ and a strong maximum at $\mathcal{R}=3$. Similar analysis for $V_{\mathrm{QH}}(\mathcal{R})$ shown in Fig. 1(a) for $8 \leq N \leq 11$ reveals the maxima at $\mathcal{R}=1$ and 5 , and the nearly vanishing $V_{\mathrm{QH}}(3)$. Actually, it follows from the comparison of Figs. 1(a) and (b) that the slightly reduced energy scale for $V_{\mathrm{QH}}(\mathcal{R})$ and the additional strongly repulsive state at $\mathcal{R}_{\mathrm{QH}}=1$ are the only significant differences between the two pseudopotentials. The $V_{\mathrm{QE}}(\mathcal{R}) \sim V_{\mathrm{QH}}(\mathcal{R}+2)$ correspondence and the fact that $V_{\mathrm{QH}}(1)$ is the largest of all $\mathrm{QE}$ or $\mathrm{QH}$ parameters will be used to construct the $\mathrm{QH}$ states corresponding to the incompressible QE states studied numerically in detail.

The above conclusions about the properties of QP-QP pseudopotentials weakly depend on such assumptions as zero layer thickness $w$ or infinite magnetic field $B$, and their oscillations at small $\mathcal{R}$ persist in realistic FQH systems. It is noteworthy that this result cannot be obtained from the literally understood original formulation of the $\mathrm{CF}$ model in which the weak "residual" $\mathrm{CF}-\mathrm{CF}$ interactions are said to result from partial cancellation of strong Coulomb and gauge interactions between the electrons. This is because these two interactions have different character and, for example, depend differently on $w$ or $B{ }^{11}$ 


\section{CORRESPONDING QE AND QH STATES}

It can be seen in Fig. 1 that $V_{\mathrm{QH}}(1)$ is the strongest anharmonic contribution to $V_{\mathrm{QH}}(\mathcal{R})$. This causes the maximum avoidance of the two-QH state with $\mathcal{R}=1$ (Laughlin $\mathrm{QH}-\mathrm{QH}$ correlations) and justifies the $\mathrm{CF}$ transformation with $2 p=2$ fluxes attached to each QH (i.e., such reapplication of the $\mathrm{CF}$ transformation to the vacancies in the partially filled CF-LL $\mathrm{L}_{0}$ ). The states of CF-QH's obtained in this way form the lowest band of $\mathrm{QH}$ states at their filling factors $\nu_{\mathrm{QH}} \leq \frac{1}{3}$. At $\nu_{\mathrm{QH}}=\frac{1}{3}$, the $\mathrm{QH}$ Laughlin state occurs that corresponds to the $\nu=\frac{2}{7}$ hierarchy/Jain state. At $\nu_{\mathrm{QH}}=\frac{1}{5}$, the CF-QH's (unlike electrons) do not bind any more vortices because of the subharmonic character of $V_{\mathrm{QH}}(\mathcal{R})$ around $\mathcal{R}=3$.

If follows from the $V_{\mathrm{QE}}(\mathcal{R}) \sim V_{\mathrm{QH}}(\mathcal{R}+2)$ correspondence seen in Fig. 1 that the pseudopotential for the interacting CF-QH's is similar to that of QE's. To confirm this, we have calculated this pseudopotential in a standard way, 8 by numerical diagonalization of $N$ QH's interacting through $V_{\mathrm{QH}}$ in a shell of angular momentum $l_{\mathrm{QH}}=\frac{3}{2}(N-1)+2$. The similarity between $V_{\mathrm{QE}}(\mathcal{R})$ and $V_{\mathrm{CF}-\mathrm{QH}}(\mathcal{R})$ not only confirms that no additional fluxes can be attached to the CF-QH's (i.e., not more than two fluxes to the original QH's), but it also implies that the same correlations will occur in QE and CF-QH systems, and that any incompressible QE state must have its CF$\mathrm{QH}$ counterpart at the same filling factor.

The conversion of the CF-QH filling factors to $\nu_{\mathrm{QH}}$ gives Eq. (1), connecting the observed states into pairs: $\nu=\left(\frac{3}{8}\right.$ and $\left.\frac{3}{10}\right),\left(\frac{4}{11}\right.$ and $\left.\frac{4}{13}\right)$, and $\left(\frac{5}{13}\right.$ and $\left.\frac{5}{17}\right)$. Using the hierarchy equation, $\nu^{-1}=2+\left(\nu^{*}\right)^{-1}$ where $\nu^{*}=1+\nu_{\mathrm{QE}}$ or $1-\nu_{\mathrm{QH}}$, it can also easily be shown that the two fractions in each pair $(\nu, \mu)$ are connected by

$$
\nu^{-1}+\mu^{-1}=6 .
$$

\section{FINITE-WIDTH EFFECT AT $\nu=\frac{6}{17}$}

While the hierarchy interpretation is certainly invalid for the three pairs of states discussed in the preceding section, and an alternative explanation must exist for their incompressibility, the situation with another observed state, $\nu=\frac{6}{17}$, corresponding to $\nu_{\mathrm{QE}}=\frac{1}{5}$, is less obvious. Its $\mathrm{QH}$ counterpart at $\nu=\frac{6}{19}$ has not been observed, and it is not clear if the finite width $w$ of the actual experimental system (which tends to weaken oscillations in $V_{\mathrm{QP}}$ ) does not lift $V_{\mathrm{QE}}(1)$ enough compared to $V_{\mathrm{QE}}(\mathcal{R} \geq 5)$ that avoiding both $\mathcal{R}=1$ and 3 at the same time (i.e., formation of the Laughlin state of the QE's with $\nu_{\mathrm{QE}}=\frac{1}{5}$ as assumed in the CF hierarchy picture) becomes energetically favorable. If true, this would be a similar scenario to that in $\mathrm{LL}_{1}$, where the $\nu=\frac{1}{3}$ state is not a Laughlin state, but the $\nu=\frac{1}{5}$ state $i s$. If the $\nu=\frac{6}{17}$ state could indeed only be observed in sufficiently wide electron systems, then it is possible that the unobserved $\nu=\frac{6}{19}$ state (corresponding to $\nu_{\mathrm{QE}}=\frac{1}{7}$ ) would simply require slightly larger width to become incompressible.

The difference between critical widths could probably be explained by the fact that $\mathrm{QH}-\mathrm{QH}$ pseudopotential parameter that must be lifted is at a larger $\mathcal{R}$ (at 3 instead of 1) which thus corresponds to a larger average in-plane QH-QH separation $\sqrt{\left\langle r^{2}\right\rangle}$. Unfortunately, our estimates of the $V_{\mathrm{QP}}(\mathcal{R})$ pseudopotentials are not sufficiently accurate to make definite predictions about the critical widths. However, the $N=10$ electron calculation for the QE's shows that $V_{\mathrm{QE}}(1)$ indeed moves up relative to $V_{\mathrm{QE}}(5)$ and $V_{\mathrm{QE}}(7)$ when the width is increased from $w=0$ to $20 \mathrm{~nm}$. Similar behavior was found for $V_{\mathrm{QH}}$ calculated for $N=8$ : the $V_{\mathrm{QH}}(3)$ moved up relative to $V_{\mathrm{QH}}(7)$ and $V_{\mathrm{QH}}(9)$ with increasing width, only at a smaller rate $d V / d w$ than it did for QE's.

\section{QP CLUSTERING}

Although in the following discussion of QP states we will concentrate on the QE's, the extension to QH's remains valid as discussed above. Even without further numerical proof it is evident from Fig. [1 alone that the QE's interacting through $V_{\mathrm{QE}}(\mathcal{R})$ will not have Laughlin correlations. This implies that the mean field $\mathrm{CF}$ transformation cannot be reapplied to the particles or vacancies in $\mathrm{CF}-\mathrm{LL}_{1}$. This rules out the simple hierarchy picture of the $\nu=\frac{4}{11}$ state, as well as the (equivalent though even less justified) interpretation involving the coexistence of CF's carrying two and four flux quanta (or vortices) $\stackrel{9}{?}^{-}$In the latter, "multi-flavor" CF model, the CF's carrying two additional flux quanta are constructed by a reapplication of the CF transformation to those QP's in the $\frac{1}{3}$-filled CF-LL ${ }_{1}$. This procedure was actually first proposed by Sitko et al., $\stackrel{10}{=}$ so it is not new, and it is equivalent to the Haldane hierarchy (except that it is expressed in terms of fermionic rather than bosonic QE's compared to Haldane's original paper $\left.{ }^{6}\right)$. Furthermore, it has been clearly demonstrated 11 in small systems with superharmonic pseudopotentials $V$ that adding $2 p=2$, $4, \ldots$ flux quanta to each particle in a mean field $\mathrm{CF}$ transformation partitions the entire many-body Hilbert space into subspaces separated by energy gaps associated with the avoided $V(\mathcal{R})$.

What are these non-Laughlin QE-QE correlations? Clearly, the avoided pair state must now be $\mathcal{R}=3$ while having pairs in the weakly repulsive $\mathcal{R}=1$ state does not increase the total interaction energy $E$ given by

$$
E=\frac{1}{2} N(N-1) \sum_{\mathcal{R}} \mathcal{G}(\mathcal{R}) V(\mathcal{R})
$$

where $\mathcal{G}(\mathcal{R})$ denotes the pair amplitude (i.e., the fraction of pairs with relative pair angular momentum $\mathcal{R}$ ). Therefore at least some of the QE's will form such pairs $\left(\mathrm{QE}_{2}\right.$ 's) or even larger clusters $\left(\mathrm{QE}_{K}\right.$ 's) at filling factors $\nu_{\mathrm{QE}}>\frac{1}{5}$ (when the avoidance of both $\mathcal{R}=1$ and 3 at 


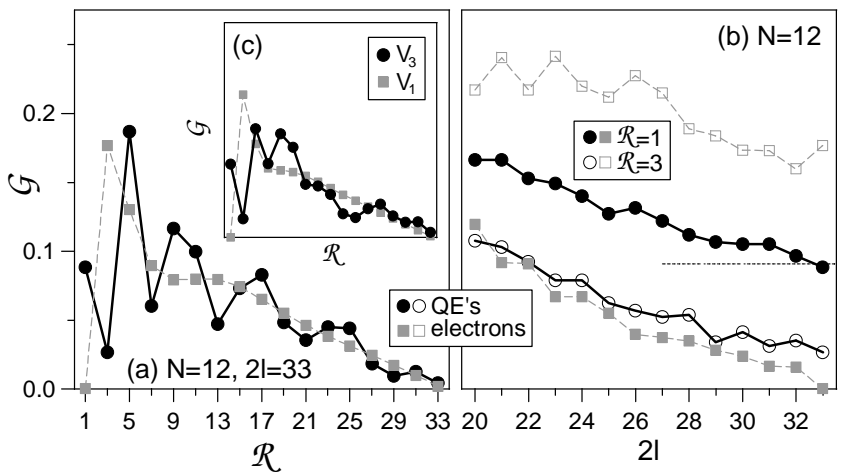

FIG. 2: (a) Pair-correlation functions (pair amplitude $\mathcal{G}$ as a function of relative pair angular momentum $\mathcal{R}$ ) for the lowest $L=0$ eigenstates of $N=12$ particles on Haldane sphere with $2 l=33$. Gray squares are for the electrons in $\mathrm{LL}_{0}$ (approximate Laughlin $\nu=\frac{1}{3}$ state) and black dots are for the QE's interacting through the pseudopotential of Fig. 11d). Inset (c) shows the same for model pseudopotentials $V_{1}$ and $V_{3}$. (b) Dependence of the two leading pair amplitudes, $\mathcal{G}(1)$ and $\mathcal{G}(3)$, on $2 l$ for $N=12$ electrons (squares) and QE's (dots). The horizontal dotted line indicates the value $\mathcal{G}=(N-1)^{-1}$ corresponding to $\frac{1}{2} N$ widely separated pairs.

the same time is not possible). Let us stress that the proposed clustering is not a result of some attractive $\mathrm{QE}-\mathrm{QE}$ interaction $\stackrel{14}{\Perp}$ but due to an obvious tendency to avoid the strongly repulsive $\mathcal{R}=3$ pair state in a system of sufficiently large density.

As an illustration for such clustering, consider a system of one-dimensional classical point charges moving along the $z$-axis, at a fixed linear density $d N / d z=1$, and interacting through a repulsive potential $V(z)$. Let us compare the following two configurations: (a) equally spread particles at $z_{k}=k$, and (b) pairs at $z_{2 k}=z_{2 k+1}=k$, where $k=0, \pm 1, \pm 2, \ldots$ The difference between the total energies counted per one particle is $\varepsilon_{b}-\varepsilon_{a}=$ $\frac{1}{2} V(0)-\sum_{k=1}^{\infty}[V(2 k-1)-V(2 k)]$, and it can have either sign depending on the form of $V(z)$. For example, if $V(z)=|z|^{-1}$ at $|z| \geq 1$, then the paired configuration (b) has lower energy if $V(z)<2 \ln 2$ at short range. For such form of $V(z)$, the transition between configurations (a) and (b) will occur at sufficiently high density $d N / d z$.

A clustered state proposed here for the QE's would be characterized by a greatly reduced pair amplitude $\mathcal{G}(3)$ compared to the Laughlin $\nu=\frac{1}{3}$ state in order to minimize the total energy. At the same time, the value of $\mathcal{G}(1)$ would be increased from nearly zero to a value of the order of $(N-1)^{-1}$ describing all $N$ QE's forming $\frac{1}{2} N$ (relatively widely separated) pairs. This behavior is demonstrated in Fig. 2(a), in which we compare $\mathcal{G}$ plotted as a function of $\mathcal{R}$, calculated for the lowest states with total angular momentum $L=0$ in systems of $N=12$ particles in the shell with $2 l=33$, interacting through different pseudopotentials. The $\mathcal{G}(\mathcal{R})$ is a form of paircorrelation function, more suitable to analyze correlations in a Hilbert space restricted to $\mathrm{LL}_{0}$ than the "real- space" pair-correlation function $\mathcal{G}(r)$. It is defined 25 in terms of the projection operator $\mathcal{P}_{i j}(\mathcal{R})$ onto the subspace in which pair $\langle i j\rangle$ is in the eigenstate $|\mathcal{R}\rangle$, and it can readily be calculated ${ }^{11}$ (using eigenfunctions of the actual pseudopotential) as an expectation value of a "selective" interaction pseudopotential $V_{\alpha}(\mathcal{R})=\delta_{\alpha \mathcal{R}}$,

$$
\mathcal{G}(\mathcal{R})=\left\langle V_{\mathcal{R}}\right\rangle .
$$

The squares in Fig. 21 a) correspond to the ground state of electrons interacting through the Coulomb potential in $\mathrm{LL}_{0}$. The full dots describe the QE's interacting through the pseudopotential shown to in Fig. I(d). In the inset (c), the squares and circles describe the ground states of selective interactions $V_{\alpha}(\mathcal{R})$. The ground state of $V_{1}$ is the exact Laughlin $\nu=\frac{1}{3}$ wavefunction, and $V_{3}$ remarkably well reproduces correlations of the QE system, which proves that it is the ability to avoid $\mathcal{R}=3$ that selects the low-energy many-QE states. The significant reduction of $\mathcal{G}(3)$ and an increase of $\mathcal{G}(1)$ when going from electrons to QE's are also clearly visible.

Since the reason for the QE clustering is the avoidance of $\mathcal{R}=3$ rather than QE-QE attraction, it seems reasonable to assume that some of the clusters should break up at lower filling factors. Mixed states of pairs and unpaired electrons have been proposed earlier in attempt to explain the $\nu=\frac{7}{3}$ state in $\mathrm{LL}_{1}, \frac{12}{\underline{2}}$ but here we have not found evidence for such behavior down to $\nu_{\mathrm{QE}}=\frac{1}{3}$. In Fig. 2(b) we plot $\mathcal{G}(1)$ and $\mathcal{G}(3)$, calculated in the lowest $L=0$ states of $N=12$ particles (electrons and QE's), as a function of $2 l$. For the QE's, as $2 l$ increases from 20 to 33 (i.e., $\nu$ decreases from $\sim \frac{1}{2}$ to $\sim \frac{1}{3}$ ), the $\mathcal{G}(3)$ decreases to zero while $\mathcal{G}(1)$ remains larger than $(N-1)^{-1}$, the value corresponding to the widely separated $\frac{1}{2} N$ pairs.

\section{INTERACTION OF QP PAIRS}

If the $\mathrm{QP}$ fluid consisted of $\mathrm{QP}_{2}$ molecules, the $\mathrm{QP}_{2}{ }^{-}$ $\mathrm{QP}_{2}$ interactions would need to be studied to understand correlations. Being pairs of fermions, the $\mathrm{QP}_{2}$ 's will be treated as bosons carrying angular momentum $l_{\mathrm{QP}_{2}}^{\text {boson }} \equiv$ $l_{\mathrm{QP}_{2}}=2 l-\mathcal{R}_{\mathrm{QP}}=2 l-1$ for the $\mathrm{QE}_{2}$ 's and $2 l-3$ for the $\mathrm{QH}_{2}$ 's. However, in two dimensions they can be easily converted to fermions by a transformation consisting of attachment of one flux quantum, 32 i.e., by an adjustment of angular momentum, $l_{\mathrm{QP}_{2}}^{\text {fermion }}=l_{\mathrm{QP}_{2}}^{\text {boson }}+\frac{1}{2}\left(N_{2}-1\right)$, where $N_{2}=\frac{1}{2} N$ is the number of pairs. The $\mathrm{QP}_{2}-\mathrm{QP}_{2}$ interaction is described by an effective pseudopotential $V_{\mathrm{QP}_{2}}\left(\mathcal{R}_{2}\right)$ that includes correlation effects caused by the fact that the two-pair wavefunction must be symmetric under exchange of $\mathrm{QP}_{2}$ bosons and at the same time antisymmetric under exchange of any two QP fermions.

In order to calculate this pseudopotential one must solve the problem of the stability of two $\mathrm{QP}_{2}$ 's in the absence of the surrounding QP's. We have done it by constructing trial paired wavefunctions $\left|\mathcal{R}_{2}\right\rangle_{\text {pair }}$ in the following way. The four QP's are divided into two pairs 


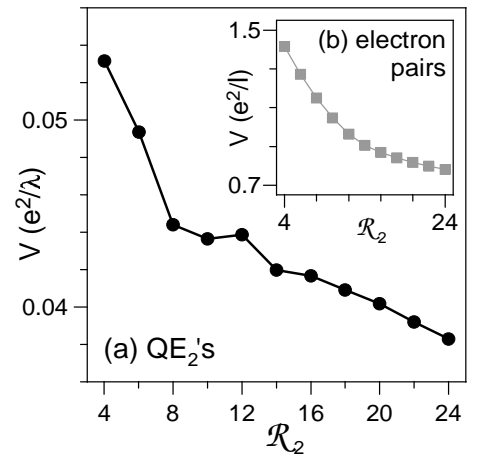

FIG. 3: Short-range parts of the pseudopotentials $V\left(\mathcal{R}_{2}\right)$ for the interaction between (a) two $\mathrm{QE}_{2}$ 's and (b) two pairs of electrons in $\mathrm{LL}_{0}$, calculated on Haldane sphere for $2 l=30$.

distinguished by two projections of pseudospin, $\sigma=\uparrow$ and $\downarrow$. A $\sigma$-asymmetric pairing interaction is defined as $V_{\sigma \sigma^{\prime}}(\mathcal{R})=-\delta_{\sigma \sigma^{\prime}} \delta_{\mathcal{R} \mathcal{R}_{\mathrm{QP}}}$ with $\mathcal{R}_{\mathrm{QE}}=1$ and $\mathcal{R}_{\mathrm{QH}}=3$. It is diagonalized in the basis of totally antisymmetric four-QP states, i.e., in the subspace of maximum total pseudospin. The resulting lowest-energy eigenstates at each angular momentum $L$ are the "maximally paired" states $\left|\mathcal{R}_{2}\right\rangle_{\text {pair }}$ corresponding to the relative angular momentum $\mathcal{R}_{2}=2 l_{\mathrm{QP}_{2}}-L$. By "maximally paired" we mean here that these states have the largest possible pair amplitude $\mathcal{G}_{\uparrow \uparrow}\left(\mathcal{R}_{\mathrm{QP}}\right)+\mathcal{G}_{\downarrow \downarrow}\left(\mathcal{R}_{\mathrm{QP}}\right)$ which is simply equal to the negative of the eigenvalue of the pairing interaction energy. The "complete pairing" corresponding to the eigenenergy equal to -2 is not allowed for identical QP's, i.e., in the subspace of maximum total pseudospin, because the three angular momenta, $\mathcal{R}_{\mathrm{QP}}$ for each pair and $\mathcal{R}_{2}$ describing relative motion of the two pairs, cannot be simultaneously conserved.

The relaxation of the angular momentum (and thus also of energy) of each of the two pairs that come in contact is due to the appropriate required symmetry of the total two-pair state with respect to an interchange of the individual QP's. This is a statistics-induced correlation effect, independent of the electric interaction between the pairs (it also occurs for the model pairing interaction that vanishes for a pair of QP's that belong to different pairs). The pair-pair pseudopotential $V_{\mathrm{QP}_{2}}\left(\mathcal{R}_{2}\right)$, calculated as the expectation energy of $V_{\mathrm{QP}}$ in the state $\left|\mathcal{R}_{2}\right\rangle_{\text {pair }}$, minus twice the energy of one pair, $2 V_{\mathrm{QP}}\left(\mathcal{R}_{\mathrm{QP}}\right)$, automatically includes this effect. However, it must be realized that the pair-pair interaction is more complicated due to the internal structure of each pair that comes into play via statistics, and that at short range its description in terms of an effective pseudopotential is only an approximation.

Fig. 31(a) shows the result obtained for the QE's in a shell with $2 l=30$, interacting through the pseudopotential of Fig. 1(d). The minimum value of $\mathcal{R}_{2}=4$ corresponds to the maximum-density four-particle droplet with $L=4 l-6$, and we only show the data up to $\mathcal{R}_{2}=24$. The $V_{\mathrm{QE}_{2}}\left(\mathcal{R}_{2}\right)$ appears weakly subharmonic at $\mathcal{R}_{2}=4$ (i.e., between $\mathcal{R}_{2}=4$ and 8 in the sense of definition
(2)), but it is clearly superharmonic at $\mathcal{R}_{2}=6$ (i.e., between $\mathcal{R}_{2}=6$ and 10). For comparison, in Fig. 3(b) we show the superharmonic and rather featureless pair-pair pseudopotential for the electrons in $\mathrm{LL}_{0}$.

\section{HALPERIN'S PAIRED QP STATES}

If QP's formed pairs ( $\mathrm{QP}_{2}$ 's) in a many-QP state, and if the pseudopotential $V_{\mathrm{QP}_{2}}\left(\mathcal{R}_{2}\right)$ were superharmonic in the entire range, then the $\mathrm{QP}_{2}$ 's would have Laughlin correlations. Being bosons, they would then form a sequence of incompressible Laughlin states at $\nu=(2 q)^{-1}$, characterized by having $\mathcal{R}_{2} \geq 2 q$ for all $\mathrm{QP}_{2}-\mathrm{QP}_{2}$ pairs. These states have been originally proposed by Halperin 16 to describe such electron states in $\mathrm{LL}_{0}$ as $\nu=\frac{2}{5}$. Later they were often invoked ${ }^{19}$ in the context of FQH effect at $\nu=\frac{5}{2}$ to describe pairing of electrons in half-filled $\mathrm{LL}_{1}$. They can be conveniently described using the following "composite boson" (CB) model ${ }^{12}$

In spherical geometry, let us consider the system of $N_{1}$ fermions (QP's) each with (integral or half-integral) angular momentum $l_{1}$ (i.e., in a LL of degeneracy $g_{1}=$ $\left.2 l_{1}+1\right)$. Neglecting the finite-size corrections, this corresponds to the filling factor $\nu_{1}=N_{1} / g_{1}$. Let the fermions form $N_{2}=\frac{1}{2} N_{1}$ bosonic pairs each with angular momentum $l_{2}=2 l_{1}-\mathcal{R}_{1}$, where $\mathcal{R}_{1}$ is an odd integer. The filling factor for the system of pairs, defined as $\nu_{2}=N_{2} / g_{2}$ where $g_{2}=2 l_{2}+1$, is given by $\nu_{2}=\frac{1}{4} \nu_{1}$. The allowed states of two bosonic pairs are labeled by total angular momentum $L=2 l_{2}-\mathcal{R}_{2}$, where $\mathcal{R}_{2}$ is an even integer.

Of all even values of $\mathcal{R}_{2}$, the lowest few are not allowed because of the Pauli exclusion principle applied to the individual fermions. The condition that the twofermion states with relative angular momentum smaller than $\mathcal{R}_{1}$ are forbidden is equivalent to the elimination of the states with $\mathcal{R}_{2} \leq 4 \mathcal{R}_{1}$ from the two-boson Hilbert space. Such a "hard core" can be accounted for by a CB transformation with $4 \mathcal{R}_{1}$ magnetic flux quanta attached to each boson ${ }^{33}$ This procedure defines the effective $\mathrm{CB}$ angular momentum $l_{2}^{*}=l_{2}-2 \mathcal{R}_{1}\left(N_{2}-1\right)$, effective LL degeneracy $g_{2}^{*}=g_{2}-4 \mathcal{R}_{1}\left(N_{2}-1\right)$, and effective filling factor $\nu_{2}^{*}=\left(\nu_{2}^{-1}-4 \mathcal{R}_{1}\right)^{-1}$.

The CB's defined in this way condense into their only allowed $l_{2}^{*}=0$ state when the corresponding fermion system has the maximum density at which pairing is still possible, $\nu_{1}=\mathcal{R}_{1}^{-1}$. At lower filling factors, the CB-LL is degenerate and the spectrum of all allowed states of the $N_{2}$ CB's represents the spectrum of the corresponding paired fermion system. In particular, using the assumption of the superharmonic form of boson-boson repulsion, condensed CB states are expected at a series of Laughlin filling factors $\nu_{2}^{*}=(2 q)^{-1}$. Here, $2 q$ is an even integer corresponding to the number of additional magnetic flux quanta attached to each $\mathrm{CB}$ in a subsequent CB transformation, $l_{2}^{*} \rightarrow l_{2}^{* *}=l_{2}^{*}-q\left(N_{2}-1\right)$, to describe Laughlin correlations between the original CB's of angular momentum $l_{2}^{*}$. From the relation between the 
fermion and CB filling factors, $\nu_{1}^{-1}=\left(4 \nu_{2}^{*}\right)^{-1}+\mathcal{R}_{1}$, we find the following sequence of fractions corresponding to Halperin's pair states, $\nu_{1}^{-1}=q / 2+\mathcal{R}_{1}$. Finally, we set $\mathcal{R}_{1}=1$ for the QE's and $\mathcal{R}_{1}=3$ for the QH's, and use the hierarchy equation,,$\frac{8}{}$

$$
\nu^{-1}=2 p+\left(1 \pm \nu_{\mathrm{QP}}\right)^{-1},
$$

to calculate the following sequences of electron filling factors $\nu$ derived from the parent $\nu=(2 p+1)^{-1}$ state

$$
\nu^{-1}=2 p+1 \mp(2+q / 2)^{-1} .
$$

In Eqs. (6) and (7), the upper sign corresponds to the QE's and the lower one to the QH's. Remarkably, all fractions reported by Pan et al. are among those predicted for the $\nu=\frac{1}{3}$ parent.

The $l_{2}^{* *}=0$ condition for the condensation of the CB's into a Laughlin $\nu_{2}^{*}=(2 q)^{-1}$ state allows the prediction of the values of $2 l \equiv 2 l_{1}$ at which these states should occur in finite systems of $N \equiv N_{1}$ QP's. The result is ${ }^{12}$

$$
2 l=\frac{q+2}{2} N-(q+1) .
$$

Interestingly, this result can be also obtained from the following picture. Let us arrange an even number of particles $(\bullet)$ in a shell by grouping them into pairs and separating each neighboring pairs by a number $q$ of empty states (०) between them (e.g., $\bullet \bullet \circ \bullet \bullet \cdots \circ \circ \bullet \bullet$ represents such paired configuration for $q=2$; note that the sequence begins and ends with a pair). Eq. (8) is then obtained by the equation of the total number of filled and empty states, $\frac{1}{2} N(q+2)-q$, with the angular momentum shell degeneracy, $2 l+1$. The success of this picture is reminiscent of a Laughlin $\nu=(2 p+1)^{-1}$ state that can be pictured as single particles separated by $2 p$ spaces (e.g., • $\diamond \circ \bullet \circ \cdots \bullet \diamond \bullet \bullet$ to represent $\nu=\frac{1}{3}$; note that different numbers of spaces correspond to an attachment of two flux quanta to a particle and to a pair).

For $q=1$ and 4, Eq. (8) gives $2 l=\frac{3}{2} N-2$ and $3 N-5$, respectively. Note the difference from $2 l=\frac{3}{2} N$ characteristic of the Jain $\nu=\frac{2}{3}$ state and and $2 l=3 N-3$ of the Laughlin $\nu=\frac{1}{3}$ state. This difference allows the distinction of Halperin's paired states from the Laughlin-Jain states based on the numerical spectra of small systems. On the other hand, $2 l=2 N-3$ predicted for $q=2$ coincides ${ }^{19}$ with the value characteristic of a Moore-Read state $\frac{18}{}$ describing a half-filled $L_{L}$. The only series of nondegenerate ground states that we found numerically in finite systems are at $2 l=2 N-3$ and $3 N-7$ (and at their particle-hole conjugate values, $2 l=2 N+1$ and $\frac{3}{2} N+2$, obtained by the replacement of $N$ by $\left.2 l+1-N\right)$.

\section{NUMERICAL RESULTS}

\section{A. Model}

Our numerical exact diagonalization calculations were carried out on Haldane sphere $\stackrel{6}{ }^{-}$In this geometry, $N$ particles are confined in a degenerate shell of angular momentum $l$. The single-particle states are labeled by $m=-l,-l+1, \ldots, l$. The two-body interaction matrix elements are connected with the pseudopotential parameters through the Clebsh-Gordan coefficients. The $N$-body interaction Hamiltonian is diagonalized numerically using a Lanczos algorithm to give the set of low energy states labeled by total angular momentum $L$.

Standard numerical calculations for $N_{e}$ electrons are not useful for studying the new observed FQH states at $\nu=\frac{3}{8}, \frac{4}{11}, \frac{5}{13}$, etc., because convincing results require values of $N_{e}$ too large to be diagonalized exactly. As these states involve pairing of Laughlin QP's and possible Laughlin correlations between the QP pairs, at least three such pairs must be considered. For $\nu=\frac{3}{8}$ this occurs for $N_{e}=14$ electrons with $2 l=33$, which seems beyond reach of exact diagonalization and explains the lack of earlier numerical evidence for incompressibility of this state. For other states, such as $\nu=\frac{4}{11}$, the systems become even larger.

Therefore, instead of diagonalizing the $N_{e}$-electron Hamiltonian, we use the QE-QE pseudopotential shown in Fig. 1(d) and diagonalize the (much smaller) interaction Hamiltonian of the $N$-QE systems. This approach is expected to accurately reproduce the low-energy spectra of interacting electrons at filling factors $\nu$ between $\frac{1}{3}$ and $\frac{2}{5}$ (up to an overall constant containing the energy of the underlying Laughlin $\nu=\frac{1}{3}$ state and the $\mathrm{QE}$ creation energies, $\varepsilon_{\mathrm{QE}}$ for each $\mathrm{QE}$ ). It is justified by fact that the $\mathrm{QE}-\mathrm{QE}$ interaction energy $V_{\mathrm{QE}}$ is small compared to the energy gap for creation of additional $\mathrm{QE}-\mathrm{QH}$ pairs, $\varepsilon_{\mathrm{QE}}+\varepsilon_{\mathrm{QH}}$. As a result, it is well-known that in this range of $\nu$, the low-energy states of (strongly interacting) electrons contain the (weakly interacting) QE's moving in an underlying (rigid) Laughlin $\nu=\frac{1}{3}$ fluid. In the CF picture, this approximation corresponds to neglecting the inter-LL excitations of CF's and only including the dynamics within the partially filled CF-LL ${ }_{1}$. In smaller systems, containing up to four QE's or QH's, this approximation has been successfully tested by direct comparison with the exact $N_{e}$-electron calculation ${ }^{11.21}$ In larger systems, it has recently been used by Lee et al ${ }^{23}$

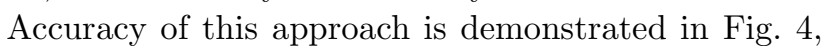
where we compare the energy spectra of two systems connected by a mean field CF transformation: (a) $N=12$ electrons in the $\mathrm{LL}_{0}$ shell with $2 l=29$ and (b) $N=4$ QE's with $2 l=9$. The four-QE energies, obtained using the pseudopotential of Fig. 11(d), are only determined up to an additive constant, but the structure and relative energies are virtually identical in the two spectra. The agreement can still be noticeably improved by using the QE-QE pseudopotential of Fig. 1(b) obtained for $N=10$ electrons (yielding the same $2 l=9$ for the pair of QE's). However, a small residual discrepancy cannot be eliminated by fitting $V_{\mathrm{QE}}(\mathcal{R})$. It is due to the fact that (although remarkably accurate) the description in terms of pair $\mathrm{QE}-\mathrm{QE}$ interactions (relying on the conservation of $\mathrm{QE}$ and $\mathrm{QH}$ numbers, i.e., on the lack of inter- 


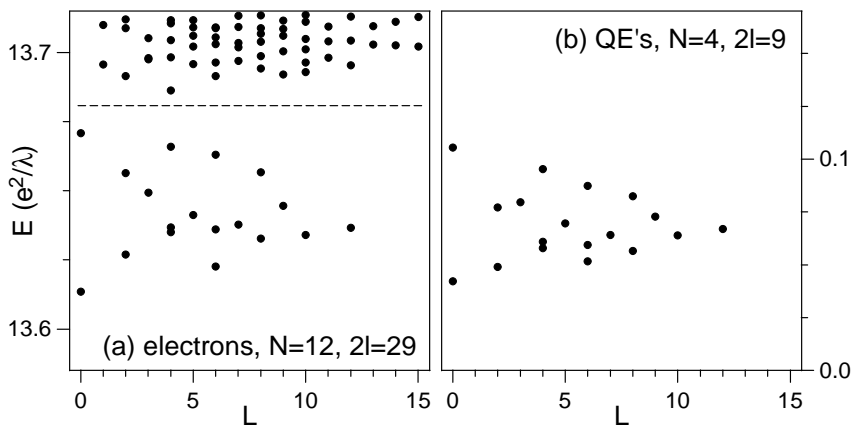

FIG. 4: Energy spectra (energy $E$ as a function of angular momentum $L$ ) calculated on Haldane sphere for $N=12$ electrons in $\mathrm{LL}_{0}$ with $2 l=29$ (a) and for $N=4$ QE's in CF-LL with $2 l=9$ (b). The energy scale is the same in both frames, but the QE spectrum is only determined up to a constant.

CF-LL excitations) is not exact. Note also that using the same pseudopotential parameters $V_{\mathrm{QE}}(\mathcal{R})$ obtained in large systems ${ }^{22}$ for the calculation of two-body interaction matrix elements at different (smaller) values of $2 l$ eliminates the finite-size effects due to surface curvature, and thus improves accuracy of the diagonalization ${ }^{34}$

Let us add the following comment about Fig. 4 Because $N=12$ electrons at $2 l=29$ have an $L=0$ ground state, and because the value of $2 l=9$ for $N=4$ QE's coincides with $3 N-3$ of a Laughlin $\nu=\frac{1}{3}$ state, this single spectrum was earlier erroneously interpreted 10.24 as a success of the CF hierarchy applied to the QE's, and this state was incorrectly assigned filling factor $\nu=\frac{4}{11}$. However, upon the analysis of correlations in this state and similar spectra of larger systems, it becomes evident that the value $2 l=9$ must be interpreted at $2 N+1$, this four-QE state is a particle-hole conjugate of the $2 N-3$ sequence, and it should be assigned QE and electron filling factors $\nu_{\mathrm{QE}}=\frac{1}{2}$ and $\nu=\frac{3}{8}$, respectively.

\section{B. Energy Spectra, Series of Incompressible Ground States, and Excitation Gaps}

We begin with a few examples of the energy spectra of up to $N=14$ QE's. Different frames in Fig. 15 show the spectra for: $N=12$ and $2 l=21$ (a), $N=10$ and $2 l=23(\mathrm{~b}), N=12$ and $2 l=27$ (c), and $N=14$ and $2 l=25(\mathrm{~d})$. Using the $\mathrm{CF}$ picture, these values of $(N, 2 l)$ can easily be converted to $N_{e}=N+(2 l-1)$ and $2 l_{e}=2(l-1)+2\left(N_{e}-1\right)$, characterizing the actual electron system (here, $2 l-1$ is the degeneracy of the completely filled CF-LL $\mathrm{L}_{0}$ with angular momentum $l-1$ ). Thus, the four $N$-QE systems in Fig. 5 correspond to: $N_{e}=32$ and $2 l_{e}=81$ (a), $N_{e}=32$ and $2 l_{e}=83(\mathrm{~b})$, $N_{e}=38$ and $2 l_{e}=99$ (c), $N_{e}=38$ and $2 l_{e}=97$ (d). It is evident that in frames (b) and (d) the FQH-like nondegenerate $(L=0)$ ground states occur, separated from the excited states by a gap $\Delta \sim 5 \cdot 10^{-3} e^{2} / \lambda$. On the other hand, in frames (a) and (c), the ground state is

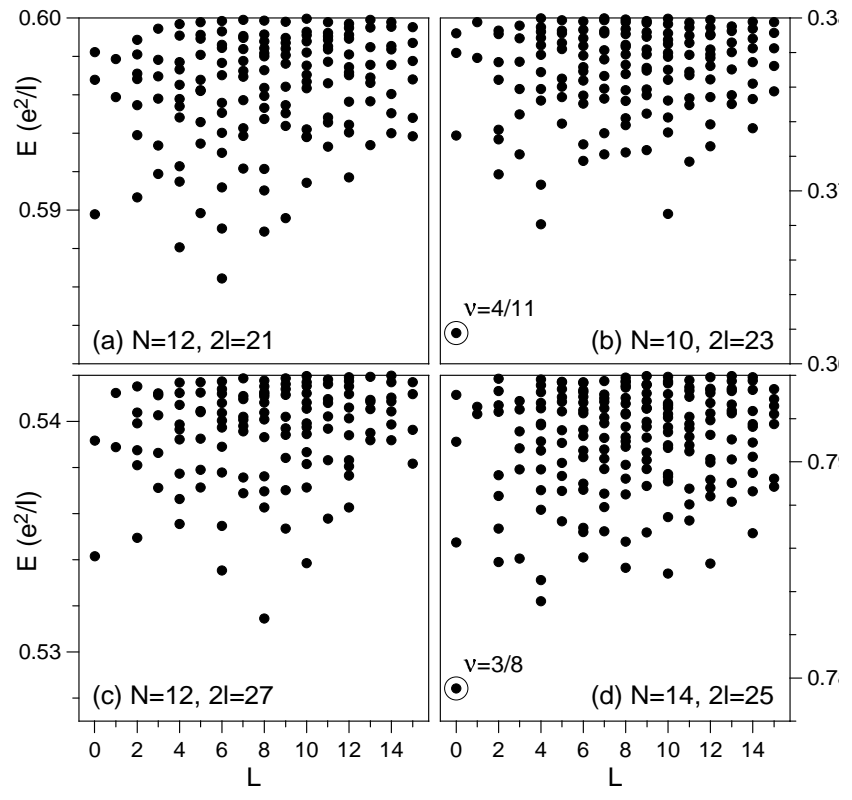

FIG. 5: Energy spectra (energy $E$ as a function of angular momentum $L$ ) of up to $N=14$ QE's in LL shells with various degeneracies $2 l+1$, calculated on Haldane sphere using QEQE interaction pseudopotential of Fig. 11(d).

degenerate $(L \neq 0)$ and no similar gap is observed. The excitation gaps $\Delta$ in (b) and (d) are larger than other energy spacings in these spectra. This indicates that they are due to the $\mathrm{QE}-\mathrm{QE}$ interactions rather than due to the size quantization in a finite system, and thus that they will not vanish in the thermodynamic $(N \rightarrow \infty)$ limit. As we show below, the $L=0$ ground states in Fig. 囵(b) and (d) correspond to $\nu=\frac{4}{11}$ and $\frac{3}{8}$ in this limit.

We have calculated similar $(N, 2 l)$ spectra for up to 14 QE's at filling factors $\nu_{\mathrm{QE}} \sim N /(2 l+1)$ between $\frac{1}{2}$ and $\frac{1}{3}$. Note that the assignment of the filling factor to a finite system $(N, 2 l)$ is not trivial and it depends on the form of correlations. The $(N, 2 l)$ sequences that correspond to a filling factor $\nu$ in the thermodynamic limit are described by a linear relation,

$$
2 l=N / \nu-\gamma_{\nu},
$$

where the "shift" $\gamma_{\nu}$ depends on the microscopic nature of the many-body state causing incompressibility at this $\nu$. For example, the sequence of finite-size nondegenerate $(L=0)$ ground states that extrapolates to $\nu=\frac{1}{3}$ occurs at $2 l=3 N-3$ for the Laughlin state, at $2 l=3 N-5$ for the Halperin paired state, 12.14 and at $2 l=3 N-7$ for the incompressible QE state identified below.

In Tab. \we present the excitation gaps obtained for the QE systems with various values of $N$ and $2 l$. The table is symmetric under the replacement of $N$ by $2 l+1-N$ which reflects the particle-hole symmetry in a partially filled QE shell (i.e., in CF-LL $L_{1}$ ). This symmetry is only approximate in real systems, but here it appears exact because of neglecting the inter-LL excitations of the CF's 
TABLE I: Excitation gaps $\Delta$, in units of $10^{-3} e^{2} / \lambda$, above the nondegenerate $(L=0)$ ground states of $N$ QE's each with angular momentum $l$, interacting through pseudopotential in Fig. 1(d). Circles (o) mark degenerate $(L \neq 0)$ ground states. The values in boldface are the largest; they all belong to the three $(N, 2 l)$ sequences corresponding to $\nu_{\mathrm{QE}}=\frac{1}{2}, \frac{1}{3}$, and $\frac{2}{3}$.

\begin{tabular}{r|ccccccccccccc}
\hline \hline$N^{2 l}$ & 17 & 18 & 19 & 20 & 21 & 22 & 23 & 24 & 25 & 26 & 27 & 28 & 29 \\
\hline 8 & $\mathbf{4 . 7 1}$ & $\circ$ & $\circ$ & $\circ$ & 0.01 & & & & & & & & \\
9 & $\circ$ & $\circ$ & $\circ$ & $\mathbf{5 . 4 7}$ & $\circ$ & $\circ$ & $\circ$ & 1.18 & & & & & \\
10 & 4.71 & $\circ$ & $\circ$ & $\circ$ & $\circ$ & $\circ$ & $\mathbf{6 . 2 9}$ & $\circ$ & 0.81 & $\circ$ & $\circ$ & & \\
11 & $\circ$ & $\circ$ & $\circ$ & $\circ$ & $\circ$ & $\circ$ & $\circ$ & $\circ$ & $\circ$ & $\mathbf{6 . 0 7}$ & $\circ$ & $\circ$ & $\circ$ \\
12 & & $\circ$ & $\circ$ & $\mathbf{5 . 4 7}$ & $\circ$ & $\circ$ & 0.37 & $\circ$ & $\mathbf{4 . 0 2}$ & $\circ$ & $\circ$ & $\circ$ & $\mathbf{5 . 2 8}$ \\
13 & & & & $\circ$ & $\circ$ & $\circ$ & $\circ$ & $\circ$ & $\circ$ & $\circ$ & $\circ$ & $\circ$ & $\circ$ \\
14 & & & & & 0.01 & $\circ$ & $\mathbf{6 . 2 9}$ & $\circ$ & $\mathbf{4 . 0 2}$ & $\circ$ & $\circ$ & $\circ$ & $\circ$ \\
15 & & & & & & & $\circ$ & $\circ$ & $\circ$ & $\circ$ & $\circ$ & $\circ$ & $\circ$ \\
16 & & & & & & & 1.18 & 0.81 & $\mathbf{6 . 0 7}$ & $\circ$ & $\circ$ & $\circ$ \\
17 & & & & & & & & & & $\circ$ & $\circ$ & $\circ$ & $\circ$ \\
18 & & & & & & & & & & $\circ$ & $\circ$ & $\mathbf{5 . 2 8}$ \\
\hline \hline
\end{tabular}

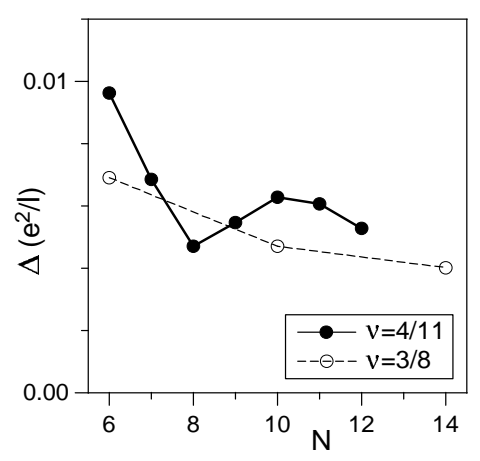

FIG. 6: Excitation gaps $\Delta$ for the $\nu_{\mathrm{QE}}=\frac{1}{3}$ series of $N$-QE ground states at $2 l=3 N-7$ (full dots) and for the $\nu_{\mathrm{QE}}=\frac{1}{2}$ series at $2 l=2 N-3$ (open circles), plotted as a function of the QE number, $N$.

in our model. The largest of the gaps $\Delta$ (those shown in boldface) occur for the following two $(N, 2 l)$ sequences: $2 l=3 N-7$ and $2 N-3$, corresponding to $\nu_{\mathrm{QE}}=\frac{1}{3}$ and $\frac{1}{2}$. Their particle-hole conjugates series (also in boldface) occur at $2 l=\frac{3}{2} N+2$ and $2 N+1$, corresponding to $\nu_{\mathrm{QE}}=1-\frac{1}{3}=\frac{2}{3}$ and $1-\frac{1}{2}=\frac{1}{2}$, respectively. Using Eq. (6), these values can be converted to the electron filling factors $\nu=\frac{3}{8}, \frac{4}{11}$, and $\frac{5}{13}$.

The dependence of the excitation gaps $\Delta$ on the QE number $N$ for the $\nu_{\mathrm{QE}}=\frac{1}{3}$ series at $2 l=3 N-7$ (full dots) and for the $\nu_{\mathrm{QE}}=\frac{1}{2}$ series at $2 l=2 N-3$ (open circles) is plotted in Fig. [6] It is difficult to accurately extrapolate our finite-size data to the thermodynamic limit to predict the magnitude of $\Delta$ in an infinite (planar) system. However, we are confident that these two series of finite-size nondegenerate ground states describe the FQH states observed experimentally at $\nu=\frac{4}{11}$ and $\frac{3}{8}$. The gaps for the larger $N$ are $\Delta \sim 5 \cdot 10^{-3} e^{2} / \lambda$. For the experimental situation of Ref. 1 (GaAs and $B=12.5 \mathrm{~T}$ ) this corresponds to $\Delta \sim 0.1 \mathrm{meV}$ or $\sim 1 K$, which seems to be a reasonable value considering the fact that the $\nu=\frac{4}{11}$ state has only been observed at temperatures as low as $T=35 \mathrm{mK}$.

\section{The $\nu_{\mathrm{QE}}=\frac{1}{3}\left(\nu=\frac{4}{11}\right)$ State}

The "shift" defined by Eq. (9) and describing the $2 l=$ $3 N-7$ sequence identified here $(\gamma=7)$ is different not only from $\gamma=3$ describing a Laughlin state, but also from $\gamma=5$ that results for Halperin's paired state (with $q=4$ ). This precludes the interpretation of these finitesize $\nu_{\mathrm{QE}}=\frac{1}{3}$ ground states found numerically (and thus also of the experimentally observed $\nu=\frac{4}{11} \mathrm{FQH}$ state) as either Laughlin or Halperin (paired) state of QE's (i.e., particles in the partially filled CF-LL 1 ). Certainly, the fact that (despite being incompressible) these states are not Laughlin states was expected from the fact that QE's form pairs over a wide range of $\nu_{\mathrm{QE}} \approx \frac{1}{3}$ (and in the whole low-energy band states, not only in the ground states). However, it is far more surprising that Halperin's paired state of QE's turns out as an invalid description for these states as well. Clearly, the correlations between the QE pairs at $\nu_{\mathrm{QE}}=\frac{1}{3}$ must be of a different, non-Laughlin type, and we do not have an alternative explanation for the incompressibility of this state.

This result is consistent with the form of the $\mathrm{QE}_{2}-\mathrm{QE}_{2}$ pseudopotential shown in Fig. [3] Because $V_{\mathrm{QE}_{2}}\left(\mathcal{R}_{2}\right)$ is only superharmonic at $\mathcal{R}_{2}=6$, the only Laughlin state expected for $\mathrm{QE}_{2}$ 's could be the one in which all values of $\mathcal{R}_{2}=4$ and 6 are simultaneously avoided. This possibly valid Halperin's paired state corresponds to $q=2$ and $\nu_{\mathrm{QE}}=\frac{1}{2}$ in Eq. (77), while the $\nu_{\mathrm{QE}}=\frac{1}{3}$ state corresponds to $q=4$ and it would have to avoid all four lowest values of $\mathcal{R}_{2}=4,6,8$, and 10 , which certainly cannot be expected from the form of $V_{\mathrm{QE}_{2}}\left(\mathcal{R}_{2}\right)$.

While we do not completely understand the correlations between QE pairs at $\nu_{\mathrm{QE}}=\frac{1}{3}$, it may be noteworthy that the value of $\gamma=7$ appropriate for the series of incompressible states found here can be obtained for the Laughlin state of $\mathrm{QE}$ triplets $\left(\mathrm{QE}_{3}\right.$ 's), each with the maximum allowed angular momentum, $L=3 l-3$. Such state would be pictured as $\bullet \bullet \bullet \circ 00000 \bullet \bullet \bullet \cdots, 000000 \bullet \bullet \bullet$ with each two closest $\mathrm{QE}$ triplets separated by six vacancies. The idea of particles grouping into triplets or larger clusters has been studied in more detail by Read and Rezayi 17 in the context of electrons in $\mathrm{LL}_{1}$. Although we do not yet have enough evidence for such particular grouping of QE's, let alone for Laughlin correlations between the clusters, this possibility is definitely worth further investigation, especially for the predicted exotic (parafermion) statistics of the excitations of such hypothetical ground state 17 Note, however, that the numerical results show an $L=0$ ground state at $2 l=3 N-7$ for every integral value of $N$, which seems inconsistent with the idea of complete clustering of QE's into molecules of any size. It can also be noticed that partial pairing with 
$\frac{1}{3} N$ of QE pairs and $\frac{1}{3} N$ of unpaired QE's also leads to $2 l=3 N-7$, but again, only for values of $N$ that are divisible by three.

\section{The $\nu_{\mathrm{QE}}=\frac{1}{2}\left(\nu=\frac{3}{8}\right)$ State}

The other sequence of finite-size $L=0$ ground states identified in Tab. [ occurs at $2 l=2 N-3$, i.e., at the same value as for the Moore-Read states of electrons halffilling $\mathrm{LL}_{1} \stackrel{18.19 .20}{\underline{10}}$ This value also coincides $\underline{12}$ with the value predicted for Halperin's paired state with $q=2$, in which the eigenstates of two $\mathrm{QE}_{2}$ bosonic pairs corresponding to the two lowest values of $\mathcal{R}_{2}=4$ and 6 , are avoided. Because of the subharmonic behavior of $V_{\mathrm{QE}_{2}}\left(\mathcal{R}_{2}\right)$ at $\mathcal{R}_{2} \neq 6$ (see Fig. [3), this $q=2$ state is the only Halperin paired state of the series given by Eq. (77) that might possibly occur in a QE system.

However, despite the facts that this sequence occurs at the predicted value of $2 l=2 N-3$ and only for even numbers of QE's (as expected for paired states), its interpretation as a Halperin paired state (or Moore-Read state) of QE's turns out incorrect. First indication is that it only seems to occur for odd numbers of QE pairs, $\frac{1}{2} N=3,5$, and 7 , while the ground states for $\frac{1}{2} N=4$ and 6 (at $2 l=13$ and 21 , respectively) both turn out degenerate. Unfortunately, we do not have data for $\frac{1}{2} N>7$ to confirm our expectation that the finite-size ground states at $2 l=2 N-3$ have $L=0$ and a large excitation gap for all odd values of $\frac{1}{2} N$. Note also that the state found here for $N=10$ and $2 l=17$ happens to be a particle-hole conjugate state of $N=8$ QE's at the same value $2 l$ (i.e., it belongs to the $2 l=\frac{3}{2} N+2$ sequence), and thus we only find two $L=0$ ground states $(N=6$ and 14) that are unique for the $2 N-3$ series.

More direct proof for the $\nu_{\mathrm{QE}}=\frac{1}{2}$ state not being Halperin's paired state (or a related Moore-Read state) comes from the analysis of its three-body correlations ${ }^{35}$ We find significant occupation of the compact triplet state $\mathrm{QE}_{3}$ with the minimum allowed relative angular momentum $\mathcal{T}=3$ at $\nu_{\mathrm{QE}}=\frac{1}{2}$, which is inconsistent with the picture of Laughlin-correlated (i.e., spatially separated) pairs. This is in contrast with the behavior of the Moore-Read paired state (an exact trial state that describes Halperin-like pairing at a half-filling) that is characterized by having exactly zero occupation of the $\mathcal{T}=3$ triplet state 19 In fact, we have calculated squared overlaps $\zeta$ of the finite-size $\nu_{\mathrm{QE}}=\frac{1}{2}$ states with the MooreRead states of QE's and they turn out very small (e.g., $\zeta=0.03$ for $N=14$ ) and insensitive to the parity of $\frac{1}{2} N$. Nevertheless, despite the fact that we do not yet understand the correlations in the $\nu_{\mathrm{QE}}=\frac{1}{2}$ state (e.g., the importance of $\frac{1}{2} N$ being odd in finite systems), we believe that the $2 l=2 N-3$ series identified here indeed describes the observed $\nu=\frac{3}{8} \mathrm{FQH}$ state.

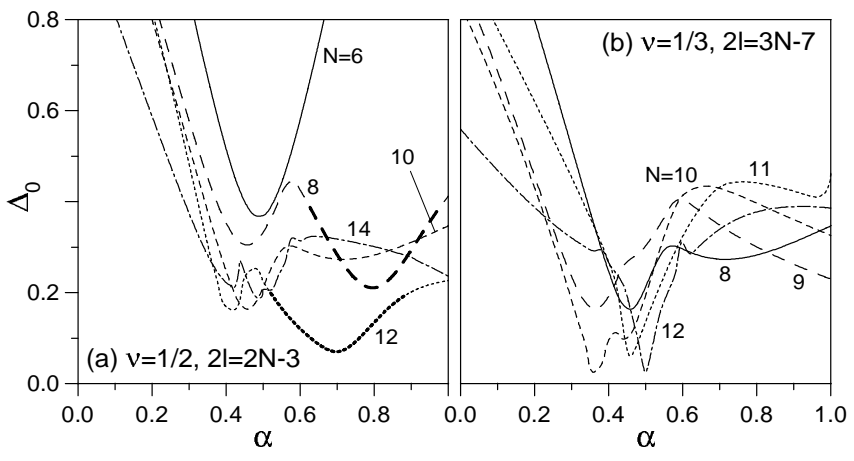

FIG. 7: The excitation gap $\Delta_{0}$ between the lowest and the first excited states in the $L=0$ subspace of $N$ particles on Haldane sphere with the values of $2 l$ corresponding to $\nu=\frac{1}{2}$ (a) and $\nu=\frac{1}{3}$ (b), plotted as a function of the interaction parameter $\alpha$ defined by Eq. (10).

\section{RESULTS FOR MODEL INTERACTIONS}

In this section we present the results of similar calculations, obtained using a model pseudopotential $U_{\alpha}(\mathcal{R})$ instead of $V_{\mathrm{QE}}(\mathcal{R})$. Its only non-vanishing coefficients are

$$
\begin{aligned}
& U_{\alpha}(1)=1-\alpha, \\
& U_{\alpha}(3)=\alpha / 2 .
\end{aligned}
$$

It is known 12 that the correlations characteristic of electrons in the partially filled $\mathrm{LL}_{0}$ and $\mathrm{LL}_{1}$ are accurately reproduced by $U_{\alpha}$ with $\alpha \approx 0$ and $\frac{1}{2}$, respectively. Similarly, by the comparison of pair amplitudes, we have confirmed that $U_{\alpha}$ with $\alpha \approx 1$ causes correlations characteristic of QE's in their partially filled LL.

We have repeated the diagonalization of a few finite systems with $2 l=2 N-3$ and $3 N-7$, for $\alpha$ varying between 0 and 1 , in order to answer the following two questions. First, to what extent is the stability of the identified $\nu=\frac{3}{8}$ and $\frac{4}{11}$ states affected by the (widthdependent) details of the QE-QE interaction? And second, does a phase transition occur for values of $\alpha$ between $\frac{1}{2}$ and 1 , indicating a different origin of the incompressibility of the $\nu=\frac{3}{8}$ and $\frac{4}{11}$ states and their electron counterparts (in $\mathrm{LL}_{1}$ ) at $\nu=\frac{5}{2}$ and $\frac{7}{3}$ ? The latter question is naturally motivated by our two observations: (i) the $2 l=2 N-3$ sequence of nondegenerate ground states occurs only for odd numbers of QE pairs $\left(\frac{1}{2} N=3,5\right.$, and 7 ), in contrast to the situation in $\mathrm{LL}_{1}$ where they occurred for any value of $\frac{1}{2} N$, and (ii) the $\nu_{\mathrm{QE}}=\frac{1}{2}$ has small overlap with the Moore-Read state (of QE's).

In Fig. [7 we plot the $L=0$ excitation energy gap $\Delta_{0}$ (difference between the two lowest energy levels at $L=0)$, as a function of $\alpha$. A minimum in $\Delta_{0}(\alpha)$ signals a (forbidden) level crossing, i.e., a phase transition in the $L=0$ subspace. Such minima occur near $\alpha=\frac{1}{2}$ for all values of $N$ and for both $2 l=2 N-3$ and $3 N-7$. They reveal destruction of Laughlin correlations that occur for small $\alpha$ (e.g., for electrons in $\mathrm{LL}_{0}$ ) and formation of in- 


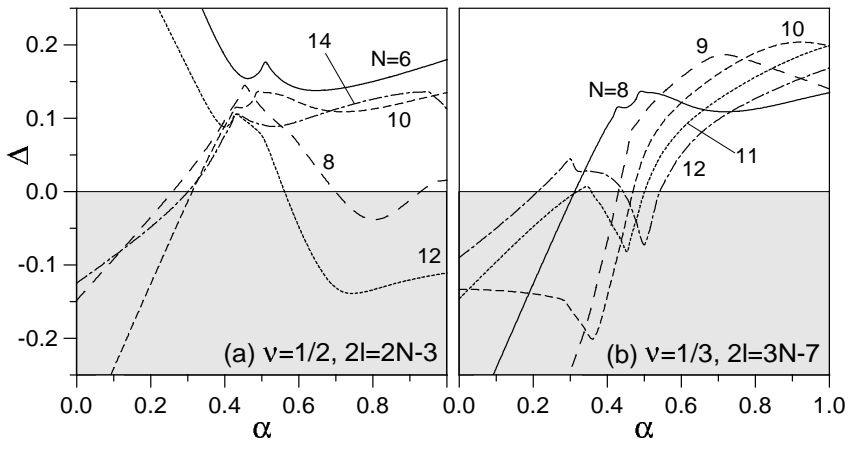

FIG. 8: The excitation gap $\Delta$ from the lowest state with $L=0$ to the lowest state with $L \neq 0$ for $N$ particles on Haldane sphere with the values of $2 l$ corresponding to $\nu=\frac{1}{2}$ (a) and $\nu=\frac{1}{3}$ (b), plotted as a function of the interaction parameter $\alpha$ defined by Eq. (10).

compressible $\nu=\frac{1}{2}$ and $\frac{1}{3}$ states of a different (paired) character that occur for $\alpha \approx \frac{1}{2}$ (e.g., for electrons in $\mathrm{LL}_{1}$ ).

In Fig. [7(a), similar strong minima occur at $\alpha \approx 0.7$ for $N=8$ and 12 (marked with thick lines). This is consistent with our observation that the correlations between the QE's and between the electrons in $\mathrm{LL}_{1}$ (both at the half-filling) are different. In Fig. (7) a) and (b), additional weaker minima between $\alpha=\frac{1}{2}$ and 1 appear also for other combinations of $N$ and $2 l$. This confirms that the $\nu=\frac{1}{2}$ and $\frac{1}{3}$ incompressible states of QE's are generally different from those of the electrons in $\mathrm{LL}_{1}$, despite the fact that they both usually occur at the same values of $2 l=2 N-3$ and $3 N-7$ in the finite systems.

The absolute excitation gaps $\Delta(\alpha)$ of the $L=0$ ground states (difference between the lowest energies at $L \neq 0$ and $L=0)$ are shown in Fig. [8] The negative value of $\Delta$ means that the absolute ground state is degenerate (i.e., has $L \neq 0)$, and the abrupt changes in the slope of $\Delta(\alpha)$ occur whenever level crossings occur for the lowest $L \neq 0$ state. Clearly, except for $N=8$ and 12 with $2 l=2 N-3$, the lowest $L=0$ states remain the absolute ground states of the system in the whole range of $\alpha$ between $\frac{1}{2}$ and 1 . This was first noticed by Greiter et al $\stackrel{19}{=}$ for $N=10$ at half-filling, and it implies that the incompressibility of the $\nu_{\mathrm{QE}}=\frac{1}{2}$ and $\frac{1}{3}$ ground states will not be easily destroyed in experimental systems by a minor deviation from the model QE-QE pseudopotential used here in the numerical diagonalization.

Let us finally examine the dependence of the leading pair amplitudes, $\mathcal{G}(1)$ and $\mathcal{G}(3)$, on $\alpha$. In Fig. 9 we plot the number of pairs $\mathcal{N}(\mathcal{R})=\frac{1}{2} N(N-1) \mathcal{G}(\mathcal{R})$, divided by $N$. A transition from Laughlin correlations at $\alpha \sim 0$ to pairing at $\alpha \sim \frac{1}{2}$ (and possibly grouping into larger clusters at $\alpha \sim 1$ ) is clearly visible in each curve. It is also confirmed that just as the Laughlin ground state remains virtually insensitive to the exact form of the interaction pseudopotential $V_{e}$ as long as it is strongly superharmonic at short range, the correlations in the $\nu_{\mathrm{QE}}=\frac{1}{2}$ and states $\frac{1}{3}$ are quite independent of the details of the $\mathrm{QE}-\mathrm{QE}$ in-

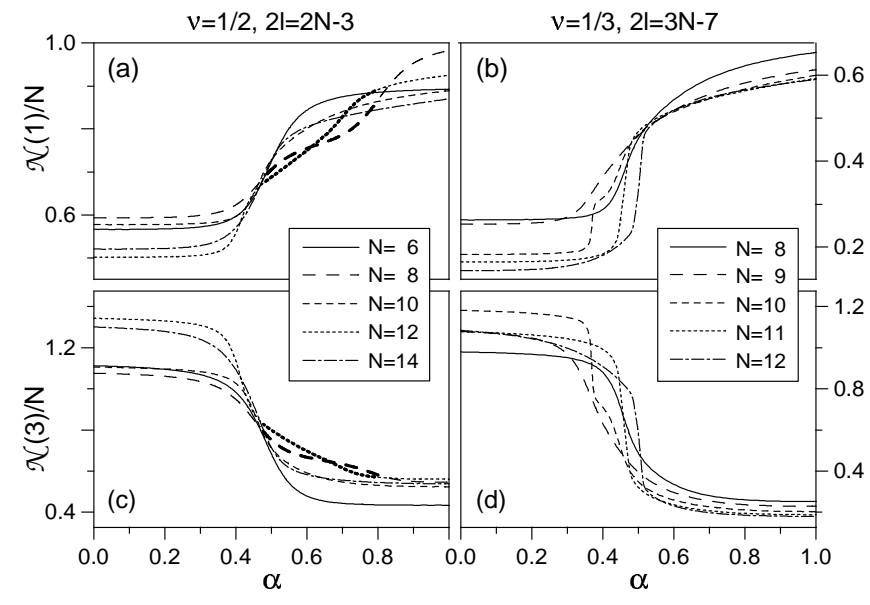

FIG. 9: The average number of pairs with relative angular momentum $\mathcal{R}=1$ (a,b) and $\mathcal{R}=3(\mathrm{c}, \mathrm{d})$ per particle, $\mathcal{N}(\mathcal{R}) / N$, calculated for the lowest state in the $L=0$ subspace of $N$ particles on Haldane sphere with the values of $2 l$ corresponding to $\nu=\frac{1}{2}(\mathrm{a}, \mathrm{c})$ and $\nu=\frac{1}{3}(\mathrm{~b}, \mathrm{~d})$, plotted as a function of the interaction parameter $\alpha$ defined by Eq. (10).

teraction, as long as $V_{\mathrm{QE}}$ is strongly subharmonic at short range. This result supports our expectation that the incompressible QE ground states found here numerically indeed describe the FQH $\nu=\frac{3}{8}$ and $\frac{4}{11}$ electron states observed in experiment.

On the other hand, correlations at $\alpha \approx \frac{1}{2}$ (electrons in $\mathrm{LL}_{1}$ ), characterized by having $\mathcal{G}(1) \approx \mathcal{G}(3)$, are quite different from those at $\alpha \sim 1$ (QE's), characterized by having the minimum possible $\mathcal{G}(3)$, much smaller than $\mathcal{G}(1)$. Finally, with thick lines in Fig. 9(a) we have marked the curves for $N=8$ and 12 in the vicinity of $\alpha \sim 0.7$ at which the forbidden crossings were found in Fig. Z(a). A different behavior of $\mathcal{N}(1) / N$ and $\mathcal{N}(3) / N$ for these two values of $N$ is clearly visible.

\section{CONCLUSIONS}

Using exact numerical diagonalization in Haldane spherical geometry, we have studied the energy spectra and wavefunctions of up to $N=14$ interacting QE's in the Laughlin $\nu=\frac{1}{3}$ parent state (i.e., CF's each carrying two flux quanta). We have demonstrated by direct calculation of the pair amplitudes $\mathcal{G}(\mathcal{R})$ that, at their sufficiently large filling factor $\left(\nu_{\mathrm{QE}}>\frac{1}{5}\right)$, the QE's form pairs or larger clusters, with a significant occupation of the minimum relative pair angular momentum, $\mathcal{R}=1$. The $\mathrm{QE}$ (and analogous QH) clustering is an opposite behavior to Laughlin correlations characterizing e.g. electrons partially filling $\mathrm{LL}_{0}$. Therefore it invalidates the reapplication of the CF picture to the individual QE's or QH's (and thus also the equivalent multi-flavor CF model) and precludes the simple hierarchy interpretation of any incompressible states at $\frac{6}{17}<\nu<\frac{2}{5}$ or $\frac{2}{7}<\nu<\frac{6}{19}$.

The series of finite-size nondegenerate ground states at 
QE filling factors $\nu_{\mathrm{QE}}=\frac{1}{2}, \frac{1}{3}$, and $\frac{2}{3}$ have been identified. These values correspond to the electronic filling factors $\nu=\frac{3}{8}, \frac{4}{11}$, and $\frac{5}{13}$, at which the FQH effect has recently been discovered $\stackrel{1}{\underline{1}}$ Due to a discussed similarity between the $\mathrm{QE}-\mathrm{QE}$ and $\mathrm{QH}-\mathrm{QH}$ interactions, these three $\mathrm{QE}$ states have their $\mathrm{QH}$ counterparts at $\nu_{\mathrm{QH}}=\frac{1}{4}, \frac{1}{5}$, and $\frac{2}{7}$, corresponding to $\nu=\frac{3}{10}, \frac{4}{13}$, and $\frac{5}{17}$, all of which have also been experimentally observed $\frac{1}{1}$ Finally, it is argued that the reported ${ }^{1} \nu=\frac{6}{17} \mathrm{FQH}$ state might be a standard hierarchy state (Laughlin $\nu_{\mathrm{QE}}=\frac{1}{5}$ state), although it could only be observed in sufficiently wide systems. Its $\mathrm{QH}$ counterpart at $\nu=\frac{6}{19}\left(\right.$ Laughlin $\nu_{\mathrm{QH}}=\frac{1}{7}$ state $)$ would require a larger width than $\nu=\frac{6}{17}$ which might explain why it has not yet been observed.

The finite-size $\nu_{\mathrm{QE}}=\frac{1}{2}, \frac{1}{3}$, and $\frac{2}{3}$ states of QE's (CF's in $\left.\mathrm{LL}_{1}\right)$ are found at the same values of $2 l=2 N-3$, $3 N-7$, and $\frac{3}{2} N+2$ as the $\nu=\frac{5}{2}$ (Moore-Read ${ }^{18.19 .20}$ ), $\frac{7}{3}$, and $\frac{8}{3} \mathrm{FQH}$ states of electrons in $\mathrm{LL}_{1}$, respectively, despite the different electron and CF pseudopotentials. Therefore we have studied the dependence of the wavefunctions and stability of the novel FQH states on the exact form of interaction at short range. We found several indications that the novel QE states are distinctly different from the electron states in $\mathrm{LL}_{1}$ : (i) the $\nu_{\mathrm{QE}}=\frac{1}{2}$ state appears incompressible only for the odd values of $\frac{1}{2} N$; (ii) the pair-correlation functions $\mathcal{G}(\mathcal{R})$ (and, especially, the triplet-correlation functions ${ }^{35}$ ) are quite different; (iii) although they remain incompressible, the ground states appear to undergo phase transitions when the $\mathrm{QE}-\mathrm{QE}$ pseudopotential is continuously transformed into that of electrons in $\mathrm{LL}_{1}$; (iv) the overlaps with the electron states in $\mathrm{LL}_{1}$ and with the Moore-Read trial state are very small. However, further studies are needed to understand these transitions. On the other hand, weak dependence of the wavefunctions and excitation gaps of the novel FQH states on the details of the $\mathrm{QE}-\mathrm{QE}$ interaction (as long as it remains strongly subharmonic at short range) justifies the use of a model pseudopotential in the realistic numerical calculation.

We have also explored Halperin's idea ${ }^{14.16}$ of the formation of Laughlin states of QE pairs ( $\mathrm{QE}_{2}$ 's). An appropriate composite boson model has been formulated and shown to predict a family of novel FQH states at a series of fractions including all those observed in experiment. However, several observations strongly point against this simple model: (i) the $\mathrm{QE}_{2}-\mathrm{QE}_{2}$ interaction pseudopotential is not superharmonic to support Laughlin correlations of $\mathrm{QE}_{2}$ 's (except possibly for $\nu_{\mathrm{QE}}=\frac{1}{2}$ ); (ii) the values of $2 l$ predicted for finite $N$ are different from these obtained from the numerical diagonalization (except for $\nu_{\mathrm{QE}}=\frac{1}{2}$ ); (iii) the numerical results do not confirm the significance of parity of the number of QE's in finite systems (the $\nu_{\mathrm{QE}}=\frac{1}{2}$ states occur only for $N=6,10$, and 14 , and the $\nu_{\mathrm{QE}}=\frac{1}{3}$ states occur for both even and odd values of $N$ ); (iv) the analysis of three-body correlations suggests formation of clusters larger than pairs ${ }^{35}$ In fact, despite an earlier expectation, ${ }^{19}$ we find ${ }^{35}$ that Halperin's pairing idea is far more appropriate for the electrons in $\mathrm{LL}_{1}$ than for QE's in CF-LL $\mathrm{L}_{1}$.

We have not found evidence for only partial pairing (and possibly Laughlin-correlated mixed states of pairs and unpaired electrons) or grouping of QE's into larger clusters of well-defined size (and possibly Laughlin correlations between them ${ }^{17}$ ). However, further investigation of both these ideas is necessary. Also, since the experiment ${ }^{1}$ indicates complete spin polarization of the novel FQH states, here we have not studied unpolarized systems, considered in great detail in a number of earlier studies begun with the work of Park and Jain ${ }^{36}$ Finally, the connection between the QE pairing studied here and recent shot-noise experiments ${ }^{37}$ indicating bunching of QP's in Laughlin and Jain FQH states at ultra-low temperatures is not yet clear.

\section{Acknowledgments}

The authors thank V. J. Goldman, M. Shayegan, and R. G. Mani for helpful discussions. This work was supported by Grant DE-FG 02-97ER45657 of the Materials Science Program - Basic Energy Sciences of the U.S. Dept. of Energy. AW acknowledges support from Grant 2P03B02424 of the Polish KBN. KSY acknowledges support from Grant R14-2002-029-01002-0 of the KOSEF.
1 W. Pan, H. L. Störmer, D. C. Tsui, L. N. Pfeiffer, K. W. Baldwin, and K. W. West, Phys. Rev. Lett. 90, 016801 (2003).

2 The $\nu=7 / 11$ and $9 / 13$ states (particle-hole conjugates of $\nu=4 / 11$ and $4 / 13$ ) were observed earlier by V.J. Goldman and M. Shayegan [Surface Sci. 229, 10 (1990)]; the $\nu=$ 4/11 and 4/13 states were also suggested by R.G. Mani and K. von Klitzing [Z. Phys. B 100, 635 (1996)] based on a "self-similarity" of the Hall resistance curve.

3 D. C. Tsui, H. L. Störmer, and A. C. Gossard, Phys. Rev. Lett. 48, 1559 (1982).

4 R. B. Laughlin, Phys. Rev. Lett. 50, 1395 (1983).

5 J. K. Jain, Phys. Rev. Lett. 63, 199 (1989).
${ }^{6}$ F. D. M. Haldane, Phys. Rev. Lett. 51, 605 (1983).

7 B. I. Halperin, Phys. Rev. Lett. 52, 1583 (1984).

8 A. Wójs and J. J. Quinn, Phys. Rev. B 61, 2846 (2000).

9 J. H. Smet, Nature 422, 391 (2003); M. R. Peterson and J. K. Jain, cond-mat/0309291

10 P. Sitko, K.-S. Yi, and J. J. Quinn, Phys. Rev. B 56, 12417 (1997).

11 A. Wójs and J. J. Quinn, Philos. Mag. B 80, 1405 (2000); Acta Phys. Pol. A 96, 593 (1999); J. J. Quinn and A. Wójs, J. Phys.: Condens. Matter 12, R265 (2000).

12 A. Wójs, Phys. Rev. B 63, 125312 (2001); A. Wójs and J. J. Quinn, Physica E 12, 63 (2002).

${ }^{13}$ F. D. M. Haldane and E. H. Rezayi, Phys. Rev. Lett. 54, 
237 (1985).

14 A. Wójs, K.-S. Yi, and J. J. Quinn, Acta Phys. Pol. A 103, 517 (2003); J. J. Quinn, A. Wójs, and K.-S. Yi, Phys. Lett. A 318, 152 (2003).

15 M. Flohr and K. Osterloh, Phys. Rev. B 67, 235316 (2003).

16 B. I. Halperin, Helv. Phys. Acta 56, 75 (1983).

17 N. Read and E. Rezayi, Phys. Rev. B 59, 8084 (1999); V. Gurarie and E. Rezayi, Phys. Rev. B 61, 5473 (2000).

18 G. Moore and N. Read, Nucl. Phys. B 360, 362 (1991); E. H. Rezayi and F. D. M. Haldane, Phys. Rev. Lett. 84, 4685 (2000).

19 M. Greiter, X.-G. Wen, and F. Wilczek, Phys. Rev. Lett. 66, 3205 (1991); Nucl. Phys. B 374, 567 (1992).

20 R. H. Morf, Phys. Rev. Lett. 80, 1505 (1998); R. H. Morf, N. d'Ambrumenil, and S. Das Sarma, Phys. Rev. B 66, 075408 (2002); R. Morf and N. d'Ambrumenil, Phys. Rev. B 68, 113309 (2003).

21 P. Sitko, S. N. Yi, K.-S. Yi, and J. J. Quinn, Phys. Rev. Lett. 76, 3396 (1996).

22 S.-Y. Lee, V. W. Scarola, and J. K. Jain, Phys. Rev. Lett. 87, 256803 (2001).

23 S.-Y. Lee, V. W. Scarola, and J. K. Jain, Phys. Rev. B 66, 085336 (2002).

24 S. S. Mandal and J. K. Jain, Phys. Rev. B 66, 155302 (2002).

${ }^{25}$ F. D. M. Haldane, The Quantum Hall Effect, edited by R. E. Prange and S. M. Girvin, (Springer-Verlag, New York, 1987), chapter 8, pp. 303-352.

26 R. D. Cowan, The Theory of Atomic Structure and Spectra (University of California Press, Berkeley, 1981); A. de Shalit and I. Talmi, Nuclear Shell Theory (Academic Press, New York, 1963).

27 A. Wójs and J. J. Quinn, Solid State Commun. 110, 45
(1999)

28 J. J. Quinn and Jennifer J. Quinn, Phys Rev. B 68, 153310 (2003).

29 T. Morinari, Phys. Rev. B 62, 15903 (2000); P. Sitko, Physica E 9, 271 (2001).

30 A. Wójs, P. Hawrylak, and J. J. Quinn, Phys. Rev. B 60, 11661 (1999); A. Wójs, I. Szlufarska, K. S. Yi, and J. J. Quinn, Phys. Rev. B 60, R11273 (1999); A. Wójs, J. J. Quinn, and P. Hawrylak, Phys. Rev. B 62, 4630 (2000).

31 A. Wójs and J. J. Quinn, Phys. Rev. B 66, 045323 (2002); Solid State Commun. 122, 407 (2002).

32 F. Wilczek, Fractional Statistics and Anyon Superconductivity (World Scientific, 1990); G. S. Canright, S. M. Girvin, and A. Brass, Phys. Rev. Lett. 63, 2291 (1989); X. C. Xie, S. He, and S. Das Sarma, Phys. Rev. Lett. 66, 389 (1991); J. J. Quinn, A. Wójs, Jennifer J. Quinn, and A. T. Benjamin, Physica E 11, 182 (2001).

33 Jennifer J. Quinn and J. Tobiska, (unpublished); A. T. Benjamin, Jennifer J. Quinn, J. J. Quinn, and A. Wójs, J. Combinat. Theory A 95, 390 (2001).

34 S. He, F. C. Zhang, X. C. Xie, and S. Das Sarma, Phys. Rev. B 42, R11376 (1990).

${ }^{35}$ A. Wójs and J. J. Quinn, unpublished.

${ }^{36}$ K. Park and J. K. Jain, Phys. Rev. B 62, R13274 (2000); I. Szlufarska, A. Wójs, and J. J. Quinn, Phys. Rev. B 64, 165318 (2001); V. W. Scarola, J. K. Jain, and E. H. Rezayi, Phys. Rev. Lett. 88, 216804 (2002); C.-C. Chang, S. S. Mandal, and J. K. Jain Phys. Rev. B 67, R121305 (2003).

37 E. Comforti, Y. C. Chung, M. Heiblum, and V. Umansky, Phys. Rev. Lett. 89, 066803 (2002); Y. C. Chung, M. Heiblum, and V. Umansky, Phys. Rev. Lett. 91, 216804 (2003). 